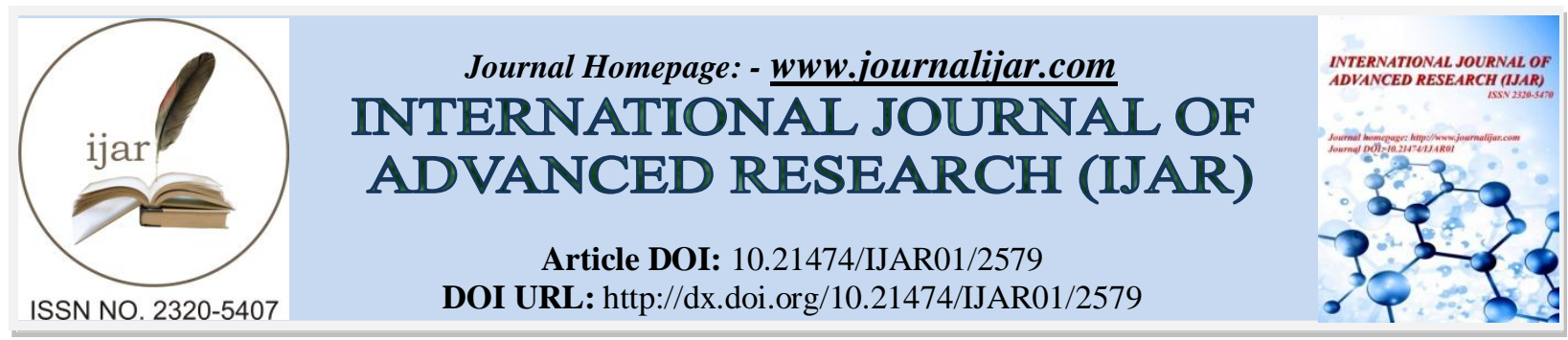

RESEARCH ARTICLE

\title{
ESTIMATION OF PARAMETERS USING LINDLEY'S METHOD.
}

\author{
A. Lavanya ${ }^{1}$ and T. Leo Alexander ${ }^{2}$. \\ 1. Research Scholar, Department of Statistics, Loyola College, Chennai-34, India \\ 2. Associate Professor, Department of Statistics, Loyola College, Chennai-34, India
}

\section{Manuscript Info}

\section{Manuscript History}

Received: 27 October 2016

Final Accepted: 25 November 2016

Published: December 2016

Key words:-

Constant Shape Bi-Weibull Distribution,

Bayesian method, Jeffreys Prior

information, Extension of Jeffreys Prior

information, Maximum Likelihood

Estimation.

\begin{abstract}
In this paper, we consider the estimation problem of the parameters of the Constant Shape Bi-Weibull Distribution based on a Failure Time data. We use the method of Maximum Likelihood and Bayesian estimation to estimate parameters. The Bayesian estimates are obtained using Lindleys approximation technique with Jeffreys Prior information and Extension of Jeffreys Prior information under three loss functions. The comparisons between different estimators are made based on simulation study and a real time data.
\end{abstract}

Copy Right, IJAR, 2016,. All rights reserved.

\section{Introduction:-}

Much of the popularity of the Weibull distribution is due to the wide variety of shapes it can assume by varying its parameters. We have considered a situation where all the units under investigation either failed before or at the end of the study. In a situation of this sort, one has at his/her disposal exact values of the observed units. In other words it is uncensored observations. We previously developed some properties of Constant Shape Bi-Weibull Distribution and Bayesian Estimation of Parameters under the Constant Shape Bi-Weibull Distribution Using Extension of Jeffreys' Prior Information under Three Loss Functions [9-15]. Now, the aim of this paper is twofold. The Maximum Likelihood Estimators of the parameters are obtained. It is observed that the MLEs cannot be obtained in closed form; we therefore propose to use the Newton-Raphson numerical approximation method to compute the MLEs via the Taylor series, and the proposed method works quite well. The second aim of this paper is to consider the Bayesian inference for parameters. In this paper, the Bayes estimates are obtained under the linear exponential (LINEX) loss, General Entropy and Squared error loss functions using Lindley's approximation technique with Jeffreys Prior information and Extension of Jeffreys Prior information. Advanced research has been done to compare MLE and that of the Bayesian approach in estimating the Weibull parameters $[4,16,20,21,2,3]$.

The rest of the paper is arranged as follows: Section 2 contains the derivative of the parameters are determined under Maximum Likelihood Estimation, In section 3 we obtained the Bayesian estimator with Jeffreys Prior information and Extension of Jeffreys Prior information under three Loss functions. Section 4 deals with simulation study and it's real time data in Section 5. Finally conclusions are provided in Section 6.

\section{Maximum likelihood estimation:-}

Suppose $T=\left(t_{1}, t_{2} \ldots t_{n}\right)$ is an uncensored observation from a sample of $n$ units or individuals under examination. Also assume that the uncensored observations (data) follow the Constant Shape Bi-Weibull model. The Constant 
Shape Bi-Weibull failure time distribution of $\sigma$ (scale parameter) and $\beta$ (shape parameter) has a probability density function (pdf) and a cumulative distribution function (cdf) as follows:

The Cumulative distribution function (CDF) is

$$
f(t)=\frac{\beta}{\sigma} t^{\beta-1} e^{-\left[\frac{t^{\beta}}{\sigma}\right]}
$$

The likelihood function is

$$
F(t)=1-e^{-\left[\frac{t^{\beta}}{\sigma}\right]} .
$$

$$
L\left(t_{i}, \sigma, \beta\right)=\prod_{i=1}^{n} \frac{\beta}{\sigma} t_{i}^{\beta-1} e^{-\left[\frac{t_{i} \beta}{\sigma}\right]} .
$$

The log-likelihood function is

$$
\ln L=n \ln \beta+(\beta-1)\left[\sum_{i=1}^{m} \ln t_{i}\right]-n \ln \sigma-\frac{1}{\sigma} \sum_{i=1}^{n} t_{i}{ }^{\beta} .
$$

By differentiating the equation (4) with respect to $\sigma$ and $\boldsymbol{\beta}$ and equating to zero, we get

and

$$
\begin{gathered}
\frac{\partial \ln L}{\partial \sigma}=-\frac{n}{\sigma}+\frac{\sum_{i=1}^{n} t_{i}{ }^{\beta}}{\sigma^{2}}=0 . \\
\frac{\partial \ln L}{\partial \beta}=\frac{n}{\beta}+\left[\sum_{i=1}^{n} \ln t_{i}\right]-\frac{1}{\sigma} \sum_{i=1}^{n} t_{i}{ }^{\beta} \ln t_{i}=0 .
\end{gathered}
$$

From equation (5), we get

$$
\hat{\sigma}=\frac{1}{n} \sum_{i=1}^{n} t_{i}{ }^{\beta} .
$$

First we shall find $\widehat{\boldsymbol{\beta}}$ and so that $\widehat{\boldsymbol{\sigma}}$ can be determined. Note that the estimators of $\sigma$ and $\beta$ are not in closed form. So that we propose to find $\widehat{\boldsymbol{\beta}}$ by using Newton-Raphson method as given below. Let $f(\boldsymbol{\beta})$ be the same as equation (6) and taking the first differential of $f(\boldsymbol{\beta})$, we have

By substituting equation (7) into equation (6), we call $f(\boldsymbol{\beta})$ as

$$
f^{\prime}(\beta)=-\left(\frac{n}{\beta^{2}}\right)-\frac{1}{\sigma} \sum_{i=1}^{n} t_{i}^{\beta}\left(\ln t_{i}\right)^{2} .
$$

Substituting equation (7) into equation (8), we obtain

$$
f(\beta)=\frac{n}{\beta}+\left[\sum_{i=1}^{n} \ln t_{i}\right]-\frac{\sum_{i=1}^{n} t_{i}{ }^{\beta} \ln t_{i}}{\frac{1}{n} \sum_{i=1}^{n} t_{i}{ }^{\beta}} .
$$

$$
f^{\prime}(\beta)=-\left\{\frac{n}{\beta^{2}}+\frac{\sum_{i=1}^{n} t_{i}^{\beta}\left(\ln t_{i}\right)^{2}}{\frac{1}{n} \sum_{i=1}^{n} t_{i}{ }^{\beta}}\right\} .
$$

Therefore, $\widehat{\boldsymbol{\beta}}$ is obtained from the equation below by carefully choosing an initial value $\boldsymbol{\beta}$ as $\boldsymbol{\beta}_{\boldsymbol{i}}$ and iterating the process till it converges:

Thus

$$
\beta_{i+1}=\beta_{i}-\frac{\frac{n}{\beta}+\left[\sum_{i=1}^{n} \ln t_{i}\right]-\frac{\sum_{i=1}^{n} t_{i} \beta \ln t_{i}}{\frac{1}{n} \sum_{i=1}^{n} t_{i}{ }^{\beta}}}{-\left\{\frac{n}{\beta^{2}+\frac{\sum_{i=1}^{n} t_{i}{ }^{\beta}\left(\ln t_{i}\right)^{2}}{\frac{1}{n} \sum_{i=1}^{n} t_{i}{ }^{2}}}\right\}}
$$

\section{Bayesian Estimation:-}

For analysing Failure time data Bayesian estimation approach has received a lot of attention. It makes use of once prior knowledge about the parameters and also takes into consideration the data available. If once prior knowledge about the parameter is available, it is suitable to make use of an informative prior but in a situation where one does not have any prior knowledge about the parameter and cannot obtain vital information from experts in this regard, then a non-informative prior will be a suitable alternative to use, Guure et al. [6]. In this study a non-informative prior approach to the parameters is employed. Given a sample $T=\left(t_{1}, t_{2} \ldots t_{n}\right)$, the likelihood (L) function follows equation (3). 
The Bayes estimators of the parameters are considered with different loss functions which are given below:

and

$$
\begin{aligned}
& \text { Linex loss: } \mathrm{L}(\hat{\theta}-\theta) \propto \exp (\mathrm{a}(\hat{\theta}-\theta))-\mathrm{a}(\hat{\theta}-\theta)-1 \\
& \text { General entropy loss: } \mathrm{L}(\hat{\theta}-\theta) \propto\left(\frac{\hat{\theta}}{\theta}\right)^{k}-k \ln \left(\frac{\hat{\theta}}{\theta}\right)-1 \\
& \text { Squared Error loss: } \mathrm{L}(\hat{\theta}-\theta) \propto(\hat{\theta}-\theta)^{2} .
\end{aligned}
$$

\section{Jeffreys Prior information:-}

Consider a likelihood function $\mathrm{L}(\theta)$, with its Fisher Information $I(\theta)=-\mathrm{E}\left(\frac{\partial^{2} \operatorname{lnL}}{\partial \beta^{2}}\right)$. The Fisher Information measures the sensitivity of an estimator. Jeffreys (1961) suggested that $\pi(\theta) \propto[I(\theta)]^{\frac{1}{2}}$ be considered as a prior for the likelihood function $\mathrm{L}(\theta)$. The Jeffreys prior is justified on the grounds of its invariance under parameterization according to Sinha [18]. Under the two-parameter Weibull distribution the non-informative (vague) prior according to Sinha and Sloan [17] is given as

$$
v(\sigma, \beta) \propto\left[\frac{1}{\sigma \beta}\right] .
$$

Let the likelihood equation which is $L\left(t_{i} \mid \sigma, \beta\right)$ be the same as (4). The joint posterior of the parameters $\sigma$ and $\boldsymbol{\beta}$ is given by

$$
\pi(\sigma, \beta \mid t) \propto \mathrm{L}(\mathrm{t} \mid \sigma, \beta) \mathrm{v}(\sigma, \beta) .
$$

The marginal distribution function is the double integral of equation (15). Therefore, the posterior probability density function of $\sigma$ and $\beta$ given the data $\left(t_{1}, t_{2}, \ldots, t_{n}\right)$ is obtained by dividing the joint posterior density function over the marginal distribution function as

$$
\pi^{*}(\sigma, \beta \mid \mathrm{t})=\frac{\mathrm{L}(\mathrm{t} \mid \sigma, \beta) \mathrm{v}(\sigma, \beta)}{\iint \mathrm{L}(\mathrm{t} \mid \sigma, \beta) \mathrm{v}(\sigma, \beta) d \sigma d \beta}
$$

Extension of Jeffreys Prior information:-

We propose a Extension of Jeffreys Prior information such that,

$$
u(\theta) \propto\left[\frac{1}{\theta}\right]^{2 c} .
$$

This is a Extension of Jeffreys Prior information, when $\mathrm{a}=1$, we have the standard Jeffreys Prior information and undefined when $\mathrm{a}=0$. Since our knowledge on the parameters is limited as a result of which a Jeffreys Prior information approach is employed on both parameters, it is important that one ensures the prior does not significantly influence the final result. If our limited or lack of knowledge influences the results, one may end-up giving wrong interpretation which could affect whatever it is we seek to address. It is as a result of this that the Extension of Jeffreys Prior information is considered.

We have,

The likelihood function from equation (3) is

$$
u(\sigma, \beta) \propto\left[\frac{1}{\sigma \beta}\right]^{2 c} .
$$

$$
L\left(t_{i} \mid \sigma, \beta\right)=\prod_{i=1}^{n} \frac{\beta}{\sigma} t_{i}^{\beta-1} e^{-\left[\frac{t_{i} \beta}{\sigma}\right]} .
$$

According to Bayes theorem, the joint posterior distribution of the parameters $\boldsymbol{\sigma}$ and $\boldsymbol{\beta}$ is

$$
\pi^{*}(\sigma, \beta \mid \mathrm{t}) \propto \mathrm{L}(\mathrm{t} \mid \sigma, \beta) \mathrm{u}(\sigma, \beta)
$$

where

$$
L\left(t_{i} \mid \sigma, \beta\right)=\frac{k}{(\sigma \beta)^{2 c}} \prod_{i=1}^{n} \frac{\beta}{\sigma} t_{i}^{\beta-1} e^{-\left[\frac{t_{i} \beta}{\sigma}\right]}
$$

and the marginal distribution is $\iint \frac{k}{(\sigma \beta)^{2 c}} \prod_{i=1}^{n} \frac{\beta}{\sigma} t_{i}^{\beta-1} e^{-\left[\frac{t_{i} \beta}{\sigma}\right]} d \sigma d \beta$,

where $k$ is the normalizing constant that makes $\pi^{*}$ a proper pdf. The posterior density function is obtained by using equation (16). 


\section{Linear Exponential (LINEX) Loss Function:-}

This loss function according to [19] rises approximately exponentially on one side of zero and approximately linearly on the other side. The sign and magnitude of the shape parameter ' $a$ ' represents the direction and degree of symmetry, respectively. There is overestimation if $a>0$ and underestimation if $a<0$ but when $\mathbf{a} \cong 0$, the LINEX Loss Function is approximately the Squared Error Loss Function. The posterior expectation of the LINEX Loss Function, according to [5], is

$$
E_{\theta} L(\hat{\theta}-\theta) \propto \exp (a \hat{\theta}) E_{\theta}(\exp (-a \theta))-a\left(\hat{\theta}-E_{\theta}(\theta)\right)-1 .
$$

The Bayes Estimator of $\boldsymbol{\theta}$, represented by $\widehat{\boldsymbol{\theta}}_{\boldsymbol{B} \boldsymbol{L}}$ under LINEX Loss Function, is the value of $\widehat{\boldsymbol{\theta}}$ which minimizes equation (20) and is given as

$$
\hat{\theta}_{B L}=-\frac{1}{a} \ln E_{\theta}(\exp (-a \theta)) .
$$

provided $E_{\theta}\left(\exp (-a \theta)\right.$ exists and is finite. The Bayes Estimator $\widehat{\boldsymbol{u}}_{\boldsymbol{B} \boldsymbol{L}}$ of a function $u=u(\exp (-a \sigma), \exp (-a \beta))$

is given as

$$
\hat{u}_{B L}=E(\exp (-a \sigma), \exp (-a \beta) \mid t)=\frac{\iint u[\exp (-a \sigma), \exp (-a \beta)] \pi^{*}(\sigma, \beta) d \sigma d \beta}{\iint \pi^{*}(\sigma, \beta) d \sigma d \beta} .
$$

From (22), it can be observed that ratio of integrals which cannot be solved analytically and for that we employ Lindley's approximation procedure to estimate the parameters.

According to Abdel-Wahid [1], Lindley proposed a ratio of integral of the form

$$
E[u(\theta) \mid x]=\frac{\int w(\theta)[L(\theta)] d \theta}{\int v(\theta)[L(\theta)] d \theta},
$$

where $\mathrm{L}(\theta)$ is the $\log$-likelihood and $\mathrm{w}(\theta)$ and $\mathrm{v}(\theta)$ are arbitrary functions of $\theta$. In applying this procedure, it is assumed that $\mathrm{v}(\theta)$ is the prior distribution for $\theta$ and $\mathrm{w}(\theta)=\mathrm{u}(\theta) \mathrm{v}(\theta)$ with $\mathrm{u}(\theta)$ being some function of interest. The posterior expectation of the Lindley approximation can be obtained from Sinha [18] and Guure and Ibrahim [7].

Taking the two parameters into consideration the following equation can be obtained

$$
\hat{\theta}=u+\frac{1}{2}\left[u_{11} \delta_{11}+u_{22} \delta_{22}\right]+u_{1} \rho_{1} \delta_{11}+u_{2} \rho_{2} \delta_{22}+\frac{1}{2}\left[L_{30} u_{1} \delta^{2}{ }_{11}+L_{03} u_{2} \delta^{2}{ }_{22}\right],
$$

where $\mathrm{L}$ is the log-likelihood equation in (4). To estimate the parameters, the following are considered and substituted into equation (24).

$$
\begin{aligned}
& u(\sigma)=\exp (-a \sigma), u_{1}=\frac{\partial u}{\partial \sigma}=-a \exp (-a \sigma), \\
& u_{11}=\frac{\partial^{2} u}{\partial^{2} \sigma}=-a^{2} \exp (-a \sigma), u_{2}=u_{22}=0, \\
& u(\beta)=\exp (-a \beta), u_{2}=\frac{\partial u}{\partial \beta}=-a \exp (-a \beta), \\
& u_{22}=\frac{\partial^{2} u}{\partial^{2} \beta}=-a^{2} \exp (-a \beta), u_{1}=u_{11}=0, \\
& \delta_{11}=\left(-L_{20}\right)^{-1}, \delta_{22}=\left(-L_{02}\right)^{-1}, \\
& L_{02}=-\left(\frac{n}{\beta^{2}}\right)-\frac{1}{\sigma} \sum_{i=1}^{n} t_{i}^{\beta}\left(\ln t_{i}\right)^{2}, \\
& L_{03}=2\left(\frac{n}{\beta^{3}}\right)-\frac{1}{\sigma} \sum_{i=1}^{n} t_{i}{ }^{\beta}\left(\ln t_{i}\right)^{3}, \\
& L_{20}=\frac{n}{\sigma^{2}}-2 \frac{\sum_{i=1}^{n} t_{i}{ }^{3}}{\sigma^{3}} \text { and } L_{30}=-2 \frac{n}{\sigma^{3}}+6 \frac{\sum_{i=1}^{n} t_{i}{ }^{\beta}}{\sigma^{4}} . \\
& \text { For Extension of Jeffreys Prior information } \\
& \rho(\sigma, \beta)=-\ln \left(\sigma^{2 c}\right)-\ln \left(\beta^{2 c}\right), \\
& \rho_{1}=\frac{\partial \rho}{\partial \sigma}=-\frac{1}{\sigma^{2 c}} \text { and } \rho_{2}=\frac{\partial \rho}{\partial \beta}=-\frac{1}{\beta^{2 c}} . \\
& \text { For Jeffreys Prior information } \\
& \rho(\sigma, \beta)=-\ln (\sigma)-\ln (\beta), \\
& \rho_{1}=\frac{\partial \rho}{\partial \sigma}=-\frac{1}{\sigma} \text { and } \rho_{2}=\frac{\partial \rho}{\partial \beta}=-\frac{1}{\beta} .
\end{aligned}
$$

\section{General Entropy Loss Function:-}

This is another useful asymmetric loss function that is used to determine whether there is overestimation or underestimation. It is a generalization of the entropy loss. 
The Bayes estimator of $\theta$, denoted by $\widehat{\boldsymbol{\theta}}_{\boldsymbol{B} \boldsymbol{G}}$ is the value of $\widehat{\boldsymbol{\theta}}$ which minimizes (13) and given as $\hat{\theta}_{B G}=\left[E_{\theta}\left(\theta^{-k}\right)\right]^{-\frac{1}{k}}$, provided $E_{\theta}\left(\theta^{-k}\right)$ exists and is finite.

The Bayes Estimator for this Loss Function is $\hat{u}_{B G}=E\left\{u\left[\sigma^{-k}, \beta^{-k}\right] \mid t\right\}=\frac{\iint u\left[\sigma^{-k}, \beta^{-k}\right] \pi^{*}(\sigma, \beta) d \sigma d \beta}{\iint \pi^{*}(\sigma, \beta) d \sigma d \beta}$.

Applying the same Lindley approach here as in (24) with $u_{1}, u_{11}$ and $u_{2}, u_{22}$ are the first and second derivatives for $\sigma$ and $\boldsymbol{\beta}$, respectively, and are given as

$u=\left[\sigma^{-k}\right], u_{1}=\frac{\partial u}{\partial \sigma}=-k\left[\sigma^{-k-1}\right]$,

$u_{11}=\frac{\partial^{2} u}{\partial^{2} \sigma}=-\left(-k^{2}-k\right) \sigma^{-k-2}$,

$u_{2}=u_{22}=0$,

$u=\left[\beta^{-k}\right], u_{2}=\frac{\partial u}{\partial \beta}=-k\left[\beta^{-k-1}\right]$,

$u_{22}=\frac{\partial^{2} u}{\partial^{2} \beta}=-\left(-k^{2}-k\right) \beta^{-k-2}$ and $u_{1}=u_{11}=0$.

\section{Symmetric Loss Function:-}

The squared error loss denotes the punishment in using $\hat{\theta}$ to estimate $\theta$ and is given by $\operatorname{L}(\hat{\theta}-\theta) \propto(\hat{\theta}-\theta)^{2}$. This loss function is symmetric in nature that is it gives equal weightage to both over and under estimation. In real life, we encounter many situations where over estimation may be more serious than under estimation or vice versa.

The Bayes Estimator $\widehat{\boldsymbol{u}}_{\boldsymbol{B} S}$ of a function $u=u(\sigma, \beta)$ of the unknown parameters under Square Error Loss Function (SELF) is the posterior mean, where

$$
\hat{u}_{B S}=E\{u[\sigma, \beta] \mid t\}=\frac{\iint u[\sigma, \beta] \pi^{*}(\sigma, \beta) d \sigma d \beta}{\iint \pi^{*}(\sigma, \beta) d \sigma d \beta} .
$$

Applying the same Lindley approach here as in (24) where $u_{1}, u_{11}$ and $u_{2}, u_{22}$ are the first and second derivatives for $\boldsymbol{\sigma}$ and $\boldsymbol{\beta}$, respectively, and are given as

$u=\sigma, u_{1}=\frac{\partial u}{\partial \sigma}=1, u_{11}=u_{2}=u_{22}=0$,

$u=\beta, u_{2}=\frac{\partial u}{\partial \beta}=1, u_{22}=u_{1}=u_{11}=0$.

\section{Simulation Study:-}

In this section, we perform a numerical study to compare the proposed estimators of parameters. Comparison among the different estimators is made with their Mean Squared Errors and Absolute Bias values. The Bayesian estimates of the parameters are derived with respect to three loss functions, which are LINEX, General Entropy and Squared error loss functions. A sample of size $\mathrm{n}=25,50$ and 100 were considered to represent relatively small, medium and large data set. The parameters were determined with Bayes using Jeffreys Prior information and Extension of Jeffreys Prior information. The values of the parameters chosen were $\sigma=0.5$ and 1.5 , and $\beta=0.8$ and 1.2. The values for the Loss parameters $(\mathbf{a}, \mathbf{k})$ are $\boldsymbol{a}=\boldsymbol{k}= \pm 0.6$ and \pm 1.6 . The loss parameters are chosen by taken into consideration the facts that they must either be below or above zero in order to determine whether the estimates obtained are under or above the actual parameters values. Also, the loss parameters of both LINEX and General entropy are chosen such that they do not respectively equal to zero (0) and negative one (-1), if they do, both loss functions turn to be approximately the Squared Error loss, Guure et al [8]. We have considered the values of Jeffreys' Extension are $\boldsymbol{c}=0.4$ and 1.4 without loss of generality. The choice of the Extension of Jeffreys Prior information is subjective since it is used to consider the proportion in which one will prefer the prior to influence the posterior density function. These were iterated 1000 times. See Calabria and Pulcini [5] for further details on how to choose the loss parameter values. Simulation results with respect to the mean squared errors and absolute errors are determined and presented for the purpose of comparison.

The Mean Squared Error and Absolute Bias given as

$$
M S E=\frac{\sum_{r=1}^{1000}\left(\hat{\theta}^{r}-\theta\right)^{2}}{R-1} \text {, and } A b s=\frac{\sum_{r=1}^{1000}\left|\hat{\theta}^{r}-\theta\right|}{R-1} .
$$

In Table 4.1 we present the Mean Square Error estimated values for the scale parameter $\sigma$ for both the MLE and Bayesian Estimation using extension of Jeffreys prior information based on the three loss functions. 
Table 4.1:- MSE Estimated Parameter $(\sigma)$ using Extension of Jeffrey's prior information.

\begin{tabular}{|c|c|c|c|c|c|c|c|c|c|c|c|c|c|}
\hline \multirow[b]{2}{*}{$n$} & \multirow[b]{2}{*}{$\sigma$} & \multirow[b]{2}{*}{$c$} & \multirow[b]{2}{*}{$\beta$} & \multirow[b]{2}{*}{$\hat{\sigma}_{M L}$} & \multirow[b]{2}{*}{$\hat{\sigma}_{B S}$} & $\hat{\sigma}_{B L}$ & $\hat{\sigma}_{B G}$ & $\hat{\sigma}_{B L}$ & $\hat{\sigma}_{B G}$ & $\hat{\sigma}_{B L}$ & $\hat{\sigma}_{B G}$ & $\hat{\sigma}_{B L}$ & $\hat{\sigma}_{B G}$ \\
\hline & & & & & & \multicolumn{2}{|c|}{$\mathrm{a}=\mathrm{k}=0.6$} & \multicolumn{2}{|c|}{$\mathrm{a}=\mathrm{k}=-0.6$} & \multicolumn{2}{|c|}{$\mathrm{a}=\mathrm{k}=1.6$} & \multicolumn{2}{|c|}{$\mathrm{a}=\mathrm{k}=-1.6$} \\
\hline \multirow[t]{8}{*}{25} & 0.5 & 0.4 & 0.8 & 0.1921 & $2.3 e-07$ & $6.0 \mathrm{e}-08$ & $3.2 \mathrm{e}-08$ & $1.1 \mathrm{e}-07$ & $9.3 e-08$ & $2.3 e-07$ & $1.8 \mathrm{e}-06$ & $1.0 \mathrm{e}-06$ & $4.3 e-07$ \\
\hline & 0.5 & 0.4 & 1.2 & 0.1861 & $2.6 \mathrm{e}-07$ & $6.8 \mathrm{e}-08$ & $4.2 \mathrm{e}-08$ & $1.2 \mathrm{e}-07$ & $1.0 \mathrm{e}-07$ & $2.6 e-07$ & $1.7 \mathrm{e}-06$ & $1.2 \mathrm{e}-06$ & $4.9 \mathrm{e}-07$ \\
\hline & 0.5 & 1.4 & 0.8 & 0.2693 & $3.8 \mathrm{e}-07$ & $6.2 \mathrm{e}-08$ & $2.7 \mathrm{e}-06$ & $2.9 \mathrm{e}-07$ & $2.9 \mathrm{e}-07$ & $1.1 \mathrm{e}-07$ & 0.00011 & $7.3 e-06$ & $2.8 \mathrm{e}-07$ \\
\hline & 0.5 & 1.4 & 1.2 & 0.1170 & $1.1 \mathrm{e}-06$ & $1.7 \mathrm{e}-07$ & $9.3 e-06$ & $9.1 \mathrm{e}-07$ & $9.3 e-07$ & $2.9 \mathrm{e}-07$ & 0.00040 & $2.3 e-05$ & $7.6 \mathrm{e}-07$ \\
\hline & 1.5 & 0.4 & 0.8 & 0.4394 & 0.0014 & 0.0001 & $8.3 e-05$ & 0.0023 & 0.0003 & $5.6 e-05$ & 0.00017 & 0.18789 & 0.0071 \\
\hline & 1.5 & 0.4 & 1.2 & 1.2181 & $3.9 \mathrm{e}-06$ & $5.1 \mathrm{e}-07$ & $1.1 \mathrm{e}-08$ & $2.4 \mathrm{e}-06$ & $6.4 \mathrm{e}-07$ & $4.2 \mathrm{e}-07$ & $1.5 \mathrm{e}-07$ & $7.5 e-05$ & $2.8 \mathrm{e}-05$ \\
\hline & 1.5 & 1.4 & 0.8 & 2.5425 & $1.8 \mathrm{e}-06$ & $2.0 \mathrm{e}-07$ & $2.7 \mathrm{e}-08$ & $1.7 \mathrm{e}-06$ & $3.5 \mathrm{e}-07$ & $1.4 \mathrm{e}-07$ & $2.2 \mathrm{e}-12$ & $3.3 e-06$ & $1.1 \mathrm{e}-05$ \\
\hline & 1.5 & 1.4 & 1.2 & 2.3345 & $2.2 \mathrm{e}-06$ & $2.3 e-07$ & $3.3 \mathrm{e}-08$ & $2.0 \mathrm{e}-06$ & $4.1 \mathrm{e}-07$ & $1.7 \mathrm{e}-07$ & $1.0 \mathrm{e}-10$ & $5.3 e-06$ & $1.3 \mathrm{e}-05$ \\
\hline \multirow[t]{8}{*}{50} & 0.5 & 0.4 & 0.8 & 1.3796 & $1.8 \mathrm{e}-08$ & $4.9 \mathrm{e}-09$ & $4.3 e-10$ & $8.2 \mathrm{e}-09$ & $6.8 \mathrm{e}-09$ & $2.0 \mathrm{e}-08$ & $4.0 \mathrm{e}-07$ & $6.8 \mathrm{e}-08$ & $3.6 \mathrm{e}-08$ \\
\hline & 0.5 & 0.4 & 1.2 & 0.5619 & $1.5 \mathrm{e}-07$ & $3.9 \mathrm{e}-08$ & $5.1 \mathrm{e}-08$ & $7.8 \mathrm{e}-08$ & $6.7 \mathrm{e}-08$ & $1.4 \mathrm{e}-07$ & $1.9 \mathrm{e}-07$ & $8.6 \mathrm{e}-07$ & $2.7 \mathrm{e}-07$ \\
\hline & 0.5 & 1.4 & 0.8 & & & & & & & & & & $4.2 \mathrm{e}-08$ \\
\hline & 0.5 & 1.4 & 1.2 & 0.3621 & $3.8 \mathrm{e}-07$ & $5.5 \mathrm{e}-08$ & $3.9 \mathrm{e}-06$ & $3.2 \mathrm{e}-07$ & $3.4 \mathrm{e}-07$ & $7.5 \mathrm{e}-08$ & 0.00018 & $9.2 \mathrm{e}-06$ & $2.1 \mathrm{e}-07$ \\
\hline & 1.5 & 0.4 & 0.8 & 3.1728 & $5.4 \mathrm{e}-06$ & $5.8 \mathrm{e}-07$ & $8.7 \mathrm{e}-08$ & $5.2 \mathrm{e}-06$ & $1.0 \mathrm{e}-06$ & $4.1 \mathrm{e}-07$ & $1.4 \mathrm{e}-09$ & $1.8 \mathrm{e}-05$ & $3.4 \mathrm{e}-05$ \\
\hline & 1.5 & 0.4 & 1.2 & 7.7845 & $2.8 \mathrm{e}-07$ & $4.2 \mathrm{e}-08$ & $2.2 \mathrm{e}-11$ & $1.1 \mathrm{e}-07$ & $4.1 \mathrm{e}-08$ & $3.8 \mathrm{e}-08$ & $4.0 \mathrm{e}-08$ & $2.6 e-05$ & $2.2 \mathrm{e}-06$ \\
\hline & 1.5 & 1.4 & 0.8 & 5.2676 & $2.1 \mathrm{e}-06$ & $2.1 \mathrm{e}-07$ & $4.8 \mathrm{e}-08$ & $6.7 \mathrm{e}-06$ & $1.1 \mathrm{e}-06$ & $3.3 e-07$ & $1.1 \mathrm{e}-07$ & 0.00016 & $3.2 \mathrm{e}-05$ \\
\hline & 1.5 & 1.4 & 1.2 & 3.6848 & $6.7 \mathrm{e}-06$ & $6.2 \mathrm{e}-07$ & $2.0 \mathrm{e}-07$ & $8.1 \mathrm{e}-06$ & $1.3 \mathrm{e}-06$ & $3.9 \mathrm{e}-07$ & $1.5 \mathrm{e}-07$ & 0.00022 & $3.8 \mathrm{e}-05$ \\
\hline \multirow[t]{8}{*}{100} & 0.5 & 0.4 & 0.8 & 3.2633 & $1.5 \mathrm{e}-08$ & $3.9 \mathrm{e}-09$ & $2.1 \mathrm{e}-09$ & $7.1 \mathrm{e}-09$ & $6.0 \mathrm{e}-09$ & $1.5 \mathrm{e}-08$ & $1.1 \mathrm{e}-07$ & $6.9 \mathrm{e}-08$ & $2.8 \mathrm{e}-08$ \\
\hline & 0.5 & 0.4 & 1.2 & & $5.6 \mathrm{e}-08$ & $1.4 \mathrm{e}-08$ & $2.3 \mathrm{e}-08$ & $2.8 \mathrm{e}-08$ & $2.5 \mathrm{e}-08$ & $5.1 \mathrm{e}-08$ & $1.3 \mathrm{e}-08$ & $3.3 e-07$ & $9.7 \mathrm{e}-08$ \\
\hline & 0.5 & 1.4 & 0.8 & 3.2164 & $3.8 \mathrm{e}-08$ & $6.2 \mathrm{e}-09$ & $2.8 \mathrm{e}-07$ & $3.0 \mathrm{e}-08$ & $3.0 \mathrm{e}-08$ & $1.1 \mathrm{e}-08$ & $1.1 \mathrm{e}-05$ & $7.6 e-07$ & $2.8 \mathrm{e}-08$ \\
\hline & 0.5 & 1.4 & 1.2 & 1.2291 & $8.8 \mathrm{e}-08$ & $1.1 \mathrm{e}-08$ & $1.1 \mathrm{e}-06$ & $8.2 \mathrm{e}-08$ & $8.7 \mathrm{e}-08$ & $1.1 \mathrm{e}-08$ & $5.8 \mathrm{e}-05$ & $2.5 \mathrm{e}-06$ & $3.8 \mathrm{e}-08$ \\
\hline & 1.5 & 0.4 & 0.8 & 18.333 & $3.5 e-07$ & $4.3 e-08$ & $1.6 \mathrm{e}-09$ & $2.3 e-07$ & $5.9 \mathrm{e}-08$ & $3.4 \mathrm{e}-08$ & $7.8 \mathrm{e}-09$ & $3.1 \mathrm{e}-06$ & $2.4 \mathrm{e}-06$ \\
\hline & 1.5 & 0.4 & 1.2 & 20.388 & $2.4 \mathrm{e}-07$ & $3.1 \mathrm{e}-08$ & $6.9 \mathrm{e}-10$ & $1.5 e-07$ & $4.0 \mathrm{e}-08$ & $2.6 e-08$ & $9.5 e-09$ & $4.7 e-06$ & $1.7 \mathrm{e}-06$ \\
\hline & 1.5 & 1.4 & 0.8 & 19.072 & $7.8 \mathrm{e}-07$ & $7.6 \mathrm{e}-08$ & $1.9 \mathrm{e}-08$ & $8.8 \mathrm{e}-07$ & $1.5 \mathrm{e}-07$ & $5.0 \mathrm{e}-08$ & $8.7 e-09$ & $1.5 \mathrm{e}-05$ & $4.6 e-06$ \\
\hline & 1.5 & 1.4 & 1.2 & 20.703 & $6.3 e-07$ & $6.2 \mathrm{e}-08$ & $1.4 \mathrm{e}-08$ & $6.9 \mathrm{e}-07$ & $1.2 \mathrm{e}-07$ & $4.1 \mathrm{e}-08$ & $5.3 e-09$ & $1.0 \mathrm{e}-05$ & $3.7 \mathrm{e}-06$ \\
\hline
\end{tabular}

ML: Maximum Likelihood, BS: Squared Error Loss function, BL: LINEX Loss function, BG: General Entropy Loss function.

In Table 4.2 we present the Mean Square Error estimated values for the scale parameter $\boldsymbol{\sigma}$ for the MLE and Bayesian Estimation using Jeffreys prior information with the three loss functions.

Table 4.2:- MSE Estimated Parameter $(\sigma)$ using Jeffrey's prior information.

\begin{tabular}{|c|c|c|c|c|c|c|c|c|c|c|c|}
\hline \multirow[b]{2}{*}{$n$} & \multirow[b]{2}{*}{$\sigma$} & \multirow[b]{2}{*}{$\beta$} & \multirow{2}{*}{$\hat{\sigma}_{B S}$} & $\hat{\sigma}_{B L}$ & $\hat{\sigma}_{B G}$ & $\hat{\sigma}_{B L}$ & $\hat{\sigma}_{B G}$ & $\hat{\sigma}_{B L}$ & $\hat{\sigma}_{B G}$ & $\hat{\sigma}_{B L}$ & $\hat{\sigma}_{B G}$ \\
\hline & & & & \multicolumn{2}{|c|}{$\mathrm{a}=\mathrm{k}=0.6$} & \multicolumn{2}{|c|}{$\mathrm{a}=\mathrm{k}=-0.6$} & \multicolumn{2}{|c|}{$\mathrm{a}=\mathrm{k}=1.6$} & \multicolumn{2}{|c|}{$a=k=-1.6$} \\
\hline \multirow[t]{8}{*}{25} & 0.5 & 0.8 & $1.7 \mathrm{e}-07$ & $4.7 \mathrm{e}-08$ & $4.7 \mathrm{e}-09$ & $7.9 \mathrm{e}-08$ & $6.6 \mathrm{e}-08$ & $1.9 \mathrm{e}-07$ & $3.8 \mathrm{e}-06$ & $6.6 e-07$ & $3.5 e-07$ \\
\hline & 0.5 & 1.2 & $2.0 \mathrm{e}-07$ & $5.4 \mathrm{e}-08$ & $7.8 \mathrm{e}-09$ & $9.2 \mathrm{e}-08$ & $7.6 \mathrm{e}-08$ & $2.1 \mathrm{e}-07$ & $3.8 \mathrm{e}-06$ & $7.8 \mathrm{e}-07$ & $4.0 \mathrm{e}-07$ \\
\hline & 0.5 & 0.8 & $8.2 \mathrm{e}-08$ & $2.3 \mathrm{e}-08$ & $6.3 e-11$ & $3.5 e-08$ & $2.8 \mathrm{e}-08$ & $9.6 \mathrm{e}-08$ & $3.2 \mathrm{e}-06$ & $2.5 \mathrm{e}-07$ & $1.7 \mathrm{e}-07$ \\
\hline & 0.5 & 1.2 & $7.5 e-07$ & $1.9 \mathrm{e}-07$ & $1.9 \mathrm{e}-07$ & $3.6 \mathrm{e}-07$ & $3.1 \mathrm{e}-07$ & $7.1 \mathrm{e}-07$ & $1.9 \mathrm{e}-06$ & $3.9 \mathrm{e}-06$ & $1.3 e-06$ \\
\hline & 1.5 & 0.8 & 0.00152 & 0.00011 & $8.8 \mathrm{e}-05$ & 0.00251 & 0.00251 & $5.8 \mathrm{e}-05$ & 0.00018 & 0.20272 & 0.00749 \\
\hline & 1.5 & 1.2 & $4.7 \mathrm{e}-06$ & $5.7 \mathrm{e}-07$ & $2.5 \mathrm{e}-08$ & $3.3 e-06$ & $8.0 \mathrm{e}-07$ & $4.5 \mathrm{e}-07$ & $8.5 e-08$ & $3.1 \mathrm{e}-05$ & $3.2 \mathrm{e}-05$ \\
\hline & 1.5 & 0.8 & $6.8 \mathrm{e}-07$ & $1.0 \mathrm{e}-07$ & $4.6 \mathrm{e}-11$ & $2.6 \mathrm{e}-07$ & $9.8 \mathrm{e}-08$ & $9.2 \mathrm{e}-08$ & $9.4 \mathrm{e}-08$ & $6.1 \mathrm{e}-05$ & $5.4 \mathrm{e}-06$ \\
\hline & 1.5 & 1.2 & $8.1 \mathrm{e}-07$ & $1.1 \mathrm{e}-07$ & $3.6 \mathrm{e}-13$ & $3.3 e-07$ & $1.1 \mathrm{e}-07$ & $1.0 \mathrm{e}-07$ & $1.0 \mathrm{e}-07$ & $6.4 \mathrm{e}-05$ & $6.4 \mathrm{e}-06$ \\
\hline \multirow[t]{8}{*}{50} & 0.5 & 0.8 & $1.3 \mathrm{e}-08$ & $3.7 \mathrm{e}-09$ & $2.4 \mathrm{e}-10$ & $5.5 \mathrm{e}-09$ & $4.4 \mathrm{e}-09$ & $1.6 \mathrm{e}-08$ & $6.9 \mathrm{e}-07$ & $3.6 \mathrm{e}-08$ & $2.9 \mathrm{e}-08$ \\
\hline & 0.5 & 1.2 & $1.2 \mathrm{e}-07$ & $3.2 \mathrm{e}-08$ & $2.1 \mathrm{e}-08$ & $5.9 \mathrm{e}-08$ & $5.0 \mathrm{e}-08$ & $1.2 \mathrm{e}-07$ & $7.4 \mathrm{e}-07$ & $5.9 \mathrm{e}-07$ & $2.3 \mathrm{e}-07$ \\
\hline & 0.5 & 0.8 & $9.0 \mathrm{e}-09$ & $2.6 \mathrm{e}-09$ & $4.6 \mathrm{e}-10$ & $3.6 \mathrm{e}-09$ & $2.9 \mathrm{e}-09$ & $1.1 \mathrm{e}-08$ & $5.8 \mathrm{e}-07$ & $2.1 \mathrm{e}-08$ & $2.0 \mathrm{e}-08$ \\
\hline & 0.5 & 1.2 & $7.1 \mathrm{e}-07$ & $1.7 \mathrm{e}-07$ & $4.6 \mathrm{e}-07$ & $3.7 \mathrm{e}-07$ & $3.3 \mathrm{e}-07$ & $6.0 \mathrm{e}-07$ & $5.8 \mathrm{e}-07$ & $4.7 \mathrm{e}-06$ & $1.1 \mathrm{e}-06$ \\
\hline & 1.5 & 0.8 & $6.2 \mathrm{e}-06$ & $6.3 \mathrm{e}-07$ & $1.1 \mathrm{e}-07$ & $6.3 \mathrm{e}-06$ & $1.1 \mathrm{e}-06$ & $4.4 \mathrm{e}-07$ & $1.4 \mathrm{e}-08$ & $4.7 \mathrm{e}-05$ & $3.7 \mathrm{e}-05$ \\
\hline & 1.5 & 1.2 & $3.5 \mathrm{e}-07$ & $4.8 \mathrm{e}-08$ & $1.8 \mathrm{e}-10$ & $1.7 \mathrm{e}-07$ & $5.3 e-08$ & $4.2 \mathrm{e}-08$ & $2.8 \mathrm{e}-08$ & $1.6 \mathrm{e}-05$ & $2.6 \mathrm{e}-06$ \\
\hline & 1.5 & 0.8 & $9.5 \mathrm{e}-07$ & $1.1 \mathrm{e}-07$ & $4.0 \mathrm{e}-09$ & $2.4 \mathrm{e}-06$ & $5.0 \mathrm{e}-07$ & $2.2 \mathrm{e}-07$ & $3.7 \mathrm{e}-09$ & $2.2 \mathrm{e}-07$ & $1.7 \mathrm{e}-05$ \\
\hline & 1.5 & 1.2 & $3.3 \mathrm{e}-06$ & $3.7 \mathrm{e}-07$ & $4.5 \mathrm{e}-08$ & $3.0 \mathrm{e}-06$ & $6.2 \mathrm{e}-07$ & $2.7 \mathrm{e}-07$ & $7.4 \mathrm{e}-10$ & $2.8 \mathrm{e}-06$ & $2.1 \mathrm{e}-05$ \\
\hline \multirow[t]{4}{*}{100} & 0.5 & 0.8 & $1.1 \mathrm{e}-08$ & $3.0 \mathrm{e}-09$ & $3.3 e-10$ & $5.1 \mathrm{e}-09$ & $4.2 \mathrm{e}-09$ & $1.2 \mathrm{e}-08$ & $2.3 \mathrm{e}-07$ & $4.3 \mathrm{e}-08$ & $2.2 \mathrm{e}-08$ \\
\hline & 0.5 & 1.2 & $4.5 \mathrm{e}-08$ & $1.1 \mathrm{e}-08$ & $1.1 \mathrm{e}-08$ & $2.2 \mathrm{e}-08$ & $1.9 \mathrm{e}-08$ & 4.3e-08 & $1.2 \mathrm{e}-07$ & $2.3 \mathrm{e}-07$ & $8.2 \mathrm{e}-08$ \\
\hline & 0.5 & 0.8 & $1.1 \mathrm{e}-08$ & $3.2 \mathrm{e}-09$ & $3.9 \mathrm{e}-10$ & $5.4 \mathrm{e}-09$ & $4.5 \mathrm{e}-09$ & $1.3 \mathrm{e}-08$ & $2.4 \mathrm{e}-07$ & $4.5 \mathrm{e}-08$ & $2.3 \mathrm{e}-08$ \\
\hline & 0.5 & 1.2 & $4.0 \mathrm{e}-07$ & $9.4 \mathrm{e}-08$ & $3.7 \mathrm{e}-07$ & $2.2 \mathrm{e}-07$ & $1.9 \mathrm{e}-07$ & $3.2 \mathrm{e}-07$ & $2.0 \mathrm{e}-06$ & $2.9 \mathrm{e}-06$ & $6.2 \mathrm{e}-07$ \\
\hline
\end{tabular}




\begin{tabular}{|l|l|l|l|l|l|l|l|l|l|l|l|}
\hline & 1.5 & 0.8 & $4.1 \mathrm{e}-07$ & $4.8 \mathrm{e}-08$ & $3.1 \mathrm{e}-09$ & $3.1 \mathrm{e}-07$ & $7.1 \mathrm{e}-08$ & $3.7 \mathrm{e}-08$ & $3.7 \mathrm{e}-09$ & $8.5 \mathrm{e}-07$ & $2.7 \mathrm{e}-06$ \\
& 1.5 & 1.2 & $2.9 \mathrm{e}-07$ & $3.5 \mathrm{e}-08$ & $1.6 \mathrm{e}-09$ & $2.0 \mathrm{e}-07$ & $5.0 \mathrm{e}-08$ & $2.8 \mathrm{e}-08$ & $5.3 \mathrm{e}-09$ & $1.9 \mathrm{e}-06$ & $2.0 \mathrm{e}-06$ \\
& 1.5 & 0.8 & $3.6 \mathrm{e}-07$ & $4.2 \mathrm{e}-08$ & $2.4 \mathrm{e}-09$ & $2.6 \mathrm{e}-07$ & $6.2 \mathrm{e}-08$ & $3.3 \mathrm{e}-08$ & $4.4 \mathrm{e}-09$ & $1.2 \mathrm{e}-06$ & $2.4 \mathrm{e}-06$ \\
& 1.5 & 1.2 & $2.8 \mathrm{e}-07$ & $3.4 \mathrm{e}-08$ & $1.4 \mathrm{e}-09$ & $1.9 \mathrm{e}-07$ & $4.7 \mathrm{e}-08$ & $2.7 \mathrm{e}-08$ & $5.5 \mathrm{e}-09$ & $2.1 \mathrm{e}-06$ & $1.9 \mathrm{e}-06$ \\
\hline
\end{tabular}

From Table 4.1 and 4.2 it is observed that Bayes estimation with General Entropy and LINEX loss function provides the smallest MSE values in most cases especially compared when the loss parameter values are $(0.6,1.6)$. Also sample size increases MLE has increased and Bayes estimation under all loss functions have decreases in MSE values.

In Table 4.3 we present the Mean Square Error estimated values for the shape parameter $\boldsymbol{\beta}$ for both the MLE and Bayesian Estimation using extension of Jeffreys prior information with the three loss functions.

Table 4.3:- MSE Estimated Parameter $(\boldsymbol{\beta})$ using Extension of Jeffrey's prior information

\begin{tabular}{|c|c|c|c|c|c|c|c|c|c|c|c|c|c|}
\hline \multirow{2}{*}{$\mathrm{n}$} & \multirow{2}{*}{$\sigma$} & \multirow{2}{*}{ c } & \multirow{2}{*}{$\beta$} & \multirow{2}{*}{$\hat{\beta}_{M L}$} & \multirow{2}{*}{$\hat{\beta}_{B S}$} & $\hat{\beta}_{B L}$ & $\hat{\beta}_{B G}$ & $\hat{\beta}_{B L}$ & $\hat{\beta}_{B G}$ & $\hat{\beta}_{B L}$ & $\hat{\beta}_{B G}$ & $\hat{\beta}_{B L}$ & $\hat{\beta}_{B G}$ \\
\hline & & & & & & \multicolumn{2}{|c|}{$\mathrm{a}=\mathrm{k}=0.6$} & \multicolumn{2}{|c|}{$\mathrm{a}=\mathrm{k}=-0.6$} & \multicolumn{2}{|c|}{$\mathrm{a}=\mathrm{k}=1.6$} & \multicolumn{2}{|c|}{$\mathrm{a}=\mathrm{k}=-1.6$} \\
\hline \multirow[t]{8}{*}{25} & 0.5 & 0.4 & 0.8 & 0.0010 & $1.3 \mathrm{e}-07$ & $7.7 \mathrm{e}-09$ & $4.7 \mathrm{e}-07$ & $2.3 \mathrm{e}-07$ & $9.7 \mathrm{e}-08$ & $7.0 \mathrm{e}-11$ & $9.4 \mathrm{e}-06$ & $1.6 \mathrm{e}-05$ & $8.1 \mathrm{e}-08$ \\
\hline & 0.5 & 0.4 & 1.2 & 0.0020 & $3.9 \mathrm{e}-07$ & $8.4 \mathrm{e}-09$ & $3.5 \mathrm{e}-07$ & $1.3 \mathrm{e}-06$ & $2.0 \mathrm{e}-07$ & $2.4 \mathrm{e}-09$ & $3.1 \mathrm{e}-06$ & 0.0002 & $4.2 \mathrm{e}-07$ \\
\hline & 0.5 & 1.4 & 0.8 & 0.0009 & $3.0 \mathrm{e}-07$ & $2.5 \mathrm{e}-08$ & $6.9 \mathrm{e}-07$ & $4.3 e-07$ & $1.8 \mathrm{e}-07$ & $9.6 \mathrm{e}-09$ & $1.2 \mathrm{e}-05$ & $2.6 \mathrm{e}-05$ & $3.0 \mathrm{e}-07$ \\
\hline & 0.5 & 1.4 & 1.2 & 0.0048 & $1.7 \mathrm{e}-07$ & $4.2 \mathrm{e}-10$ & $2.8 \mathrm{e}-07$ & $8.9 \mathrm{e}-07$ & $1.1 \mathrm{e}-07$ & $1.4 \mathrm{e}-08$ & $2.7 \mathrm{e}-06$ & 0.0002 & $5.2 \mathrm{e}-08$ \\
\hline & 1.5 & 0.4 & 0.8 & 0.0006 & $2.2 \mathrm{e}-08$ & $2.8 \mathrm{e}-12$ & $3.2 \mathrm{e}-07$ & $8.8 \mathrm{e}-08$ & $3.3 \mathrm{e}-08$ & $1.3 \mathrm{e}-08$ & $8.0 \mathrm{e}-06$ & $1.0 \mathrm{e}-05$ & $3.6 \mathrm{e}-09$ \\
\hline & 1.5 & 0.4 & 1.2 & 0.0005 & $2.2 \mathrm{e}-08$ & $3.8 \mathrm{e}-10$ & $8.0 \mathrm{e}-08$ & $2.0 \mathrm{e}-07$ & $2.2 \mathrm{e}-08$ & $1.0 \mathrm{e}-08$ & 8.6e-07 & $6.3 \mathrm{e}-05$ & $3.0 \mathrm{e}-09$ \\
\hline & 1.5 & 1.4 & 0.8 & 0.0001 & $3.5 \mathrm{e}-08$ & $1.9 \mathrm{e}-09$ & $1.2 \mathrm{e}-07$ & $6.2 \mathrm{e}-08$ & $2.5 \mathrm{e}-08$ & $2.0 \mathrm{e}-12$ & $2.5 \mathrm{e}-06$ & $4.5 \mathrm{e}-06$ & $2.0 \mathrm{e}-08$ \\
\hline & 1.5 & 1.4 & 1.2 & 0.0003 & $2.8 \mathrm{e}-09$ & $3.5 \mathrm{e}-09$ & $1.6 \mathrm{e}-08$ & $1.4 \mathrm{e}-08$ & $3.0 \mathrm{e}-10$ & $1.1 \mathrm{e}-08$ & $2.4 \mathrm{e}-07$ & $1.4 \mathrm{e}-05$ & $1.0 \mathrm{e}-07$ \\
\hline \multirow[t]{8}{*}{50} & 0.5 & 0.4 & 0.8 & 0.0006 & $8.6 \mathrm{e}-09$ & $2.6 \mathrm{e}-10$ & $4.8 \mathrm{e}-08$ & $1.9 \mathrm{e}-08$ & $7.7 e-09$ & $2.8 \mathrm{e}-10$ & $1.0 \mathrm{e}-06$ & $1.6 \mathrm{e}-06$ & $1.9 \mathrm{e}-09$ \\
\hline & 0.5 & 0.4 & 1.2 & 0.0022 & $1.0 \mathrm{e}-07$ & $2.1 \mathrm{e}-09$ & $9.5 \mathrm{e}-08$ & $3.6 \mathrm{e}-07$ & $5.3 \mathrm{e}-08$ & $7.5 \mathrm{e}-10$ & $8.3 e-07$ & $6.9 \mathrm{e}-05$ & $1.0 \mathrm{e}-07$ \\
\hline & 0.5 & 1.4 & 0.8 & 0.0011 & $4.8 \mathrm{e}-08$ & $3.7 \mathrm{e}-09$ & $1.2 \mathrm{e}-07$ & $7.2 \mathrm{e}-08$ & $3.0 \mathrm{e}-08$ & $1.0 \mathrm{e}-09$ & $2.2 \mathrm{e}-06$ & $4.5 \mathrm{e}-06$ & $4.4 \mathrm{e}-08$ \\
\hline & 0.5 & 1.4 & 1.2 & 0.0013 & $4.9 \mathrm{e}-08$ & $3.7 \mathrm{e}-10$ & $6.5 \mathrm{e}-08$ & $2.1 \mathrm{e}-07$ & $2.9 \mathrm{e}-08$ & $2.0 \mathrm{e}-09$ & $6.1 \mathrm{e}-07$ & $4.8 \mathrm{e}-05$ & $2.7 \mathrm{e}-08$ \\
\hline & 1.5 & 0.4 & 0.8 & 0.0007 & $4.1 \mathrm{e}-09$ & $8.9 \mathrm{e}-12$ & $6.9 \mathrm{e}-08$ & $1.7 \mathrm{e}-08$ & $6.7 \mathrm{e}-09$ & $3.2 \mathrm{e}-09$ & $1.7 \mathrm{e}-06$ & $2.2 \mathrm{e}-06$ & $1.3 \mathrm{e}-09$ \\
\hline & 1.5 & 0.4 & 1.2 & 0.0005 & $3.4 \mathrm{e}-09$ & $1.2 \mathrm{e}-10$ & $1.5 \mathrm{e}-08$ & $3.6 \mathrm{e}-08$ & $3.9 \mathrm{e}-09$ & $2.2 \mathrm{e}-09$ & $1.6 \mathrm{e}-07$ & $1.2 \mathrm{e}-05$ & $1.6 \mathrm{e}-09$ \\
\hline & 1.5 & 1.4 & 0.8 & 0.0002 & $2.0 \mathrm{e}-08$ & $1.2 \mathrm{e}-09$ & $6.8 \mathrm{e}-08$ & $4.9 \mathrm{e}-08$ & $2.0 \mathrm{e}-08$ & $3.0 \mathrm{e}-11$ & $1.9 \mathrm{e}-06$ & $3.5 \mathrm{e}-06$ & $1.8 \mathrm{e}-08$ \\
\hline & 1.5 & 1.4 & 1.2 & 0.0010 & $1.1 \mathrm{e}-09$ & $2.4 \mathrm{e}-09$ & $1.4 \mathrm{e}-08$ & $1.5 \mathrm{e}-08$ & $5.3 e-10$ & $8.6 \mathrm{e}-09$ & $2.0 \mathrm{e}-07$ & $1.2 \mathrm{e}-05$ & $6.9 \mathrm{e}-08$ \\
\hline \multirow[t]{8}{*}{100} & 0.5 & 0.4 & 0.8 & 0.0016 & $9.0 \mathrm{e}-09$ & $5.0 \mathrm{e}-10$ & $3.2 \mathrm{e}-08$ & $1.5 \mathrm{e}-08$ & $6.5 \mathrm{e}-09$ & $2.7 \mathrm{e}-12$ & $6.4 \mathrm{e}-07$ & $1.1 \mathrm{e}-06$ & $5.3 \mathrm{e}-09$ \\
\hline & 0.5 & 0.4 & 1.2 & 0.0025 & $2.9 \mathrm{e}-08$ & $6.7 \mathrm{e}-10$ & $2.5 \mathrm{e}-08$ & $9.8 \mathrm{e}-08$ & $1.4 \mathrm{e}-08$ & $1.2 \mathrm{e}-10$ & $2.1 \mathrm{e}-07$ & $1.8 \mathrm{e}-05$ & $3.3 \mathrm{e}-08$ \\
\hline & 0.5 & 1.4 & 0.8 & 0.0014 & $2.8 \mathrm{e}-08$ & $2.4 \mathrm{e}-09$ & $5.9 \mathrm{e}-08$ & $3.8 \mathrm{e}-08$ & $1.6 \mathrm{e}-08$ & $1.0 \mathrm{e}-09$ & $1.0 \mathrm{e}-06$ & $2.2 \mathrm{e}-06$ & $2.9 \mathrm{e}-08$ \\
\hline & 0.5 & 1.4 & 1.2 & 0.0015 & $1.2 \mathrm{e}-08$ & $7.6 \mathrm{e}-11$ & $1.7 \mathrm{e}-08$ & $5.6 \mathrm{e}-08$ & $7.5 \mathrm{e}-09$ & $6.0 \mathrm{e}-10$ & $1.6 \mathrm{e}-07$ & $1.2 \mathrm{e}-05$ & $6.0 \mathrm{e}-09$ \\
\hline & 1.5 & 0.4 & 0.8 & 0.0003 & $3.2 \mathrm{e}-10$ & $1.3 \mathrm{e}-11$ & $8.9 \mathrm{e}-09$ & $1.9 \mathrm{e}-09$ & $7.2 \mathrm{e}-10$ & $6.2 \mathrm{e}-10$ & $2.3 \mathrm{e}-07$ & $2.7 \mathrm{e}-07$ & $5.5 \mathrm{e}-10$ \\
\hline & 1.5 & 0.4 & 1.2 & 0.0008 & $1.7 \mathrm{e}-09$ & $3.2 \mathrm{e}-11$ & $6.5 \mathrm{e}-09$ & $1.6 \mathrm{e}-08$ & $1.8 \mathrm{e}-09$ & $8.3 \mathrm{e}-10$ & 7.0e-08 & $5.1 \mathrm{e}-06$ & $2.7 \mathrm{e}-10$ \\
\hline & 1.5 & 1.4 & 0.8 & 0.0005 & $8.6 \mathrm{e}-09$ & $5.3 \mathrm{e}-10$ & $2.8 \mathrm{e}-08$ & $1.4 \mathrm{e}-08$ & $6.0 \mathrm{e}-09$ & $2.2 \mathrm{e}-11$ & $5.5 \mathrm{e}-07$ & $1.0 \mathrm{e}-06$ & $5.7 \mathrm{e}-09$ \\
\hline & 1.5 & 1.4 & 1.2 & 0.0008 & $2.5 \mathrm{e}-10$ & $5.1 \mathrm{e}-10$ & $2.9 \mathrm{e}-09$ & $3.1 \mathrm{e}-09$ & $1.0 \mathrm{e}-10$ & $1.7 \mathrm{e}-09$ & $4.2 \mathrm{e}-08$ & 2.6e-06 & $1.4 \mathrm{e}-08$ \\
\hline
\end{tabular}

In Table 4.4 we present the Mean Square Error estimated values for the shape parameter $\boldsymbol{\beta}$ for both the MLE and Bayesian Estimation using Jeffreys prior information with the three loss functions.

Table 4.4:- MSE Estimated Parameter $(\boldsymbol{\beta})$ using Jeffreys prior information

\begin{tabular}{|c|c|c|c|c|c|c|c|c|c|c|c|}
\hline \multirow{2}{*}{$\mathrm{n}$} & \multirow{2}{*}{$\sigma$} & \multirow{2}{*}{$\beta$} & \multirow{2}{*}{$\hat{\beta}_{B S}$} & $\hat{\beta}_{B L}$ & \multicolumn{2}{|c|}{$\mathrm{a}=\mathrm{k}=0.6$} & \multicolumn{2}{c|}{$\mathrm{a}=\mathrm{k}=-0.6$} & \multicolumn{2}{|c|}{$\mathrm{a}=\mathrm{k}=1.6$} & \multicolumn{2}{c|}{$\mathrm{a}=\mathrm{k}=-1.6$} \\
\cline { 5 - 11 } & & & & $\hat{\beta}_{B L}$ & $\hat{\beta}_{B G}$ & $\hat{\beta}_{B L}$ & $\hat{\beta}_{B G}$ & $\hat{\beta}_{B L}$ & $\hat{\beta}_{B G}$ \\
\hline 25 & 0.5 & 0.8 & $1.5 \mathrm{e}-07$ & $9.3 \mathrm{e}-09$ & $5.0 \mathrm{e}-07$ & $2.5 \mathrm{e}-07$ & $1.0 \mathrm{e}-07$ & $3.5 \mathrm{e}-10$ & $9.8 \mathrm{e}-06$ & $1.8 \mathrm{e}-05$ & $1.0 \mathrm{e}-07$ \\
& 0.5 & 1.2 & $3.5 \mathrm{e}-07$ & $6.7 \mathrm{e}-09$ & $3.3 \mathrm{e}-07$ & $1.2 \mathrm{e}-06$ & $1.8 \mathrm{e}-07$ & $3.2 \mathrm{e}-09$ & $2.9 \mathrm{e}-06$ & 0.00024 & $3.5 \mathrm{e}-07$ \\
& 0.5 & 0.8 & $8.7 \mathrm{e}-08$ & $4.0 \mathrm{e}-09$ & $3.7 \mathrm{e}-07$ & $1.6 \mathrm{e}-07$ & $6.9 \mathrm{e}-08$ & $2.7 \mathrm{e}-10$ & $7.7 \mathrm{e}-06$ & $1.3 \mathrm{e}-05$ & $3.8 \mathrm{e}-08$ \\
& 0.5 & 1.2 & $4.7 \mathrm{e}-07$ & $9.9 \mathrm{e}-09$ & $4.3 \mathrm{e}-07$ & $1.6 \mathrm{e}-06$ & $2.4 \mathrm{e}-07$ & $3.1 \mathrm{e}-09$ & $3.7 \mathrm{e}-06$ & 0.00031 & $5.0 \mathrm{e}-07$ \\
& 1.5 & 0.8 & $3.2 \mathrm{e}-08$ & $7.7 \mathrm{e}-11$ & $3.5 \mathrm{e}-07$ & $1.0 \mathrm{e}-07$ & $4.1 \mathrm{e}-08$ & $1.0 \mathrm{e}-08$ & $8.5 \mathrm{e}-06$ & $1.1 \mathrm{e}-05$ & $4.5 \mathrm{e}-10$ \\
\hline
\end{tabular}




\begin{tabular}{|c|c|c|c|c|c|c|c|c|c|c|c|}
\hline & 1.5 & 1.2 & $1.6 \mathrm{e}-08$ & $6.7 \mathrm{e}-10$ & $7.4 \mathrm{e}-08$ & $1.7 \mathrm{e}-07$ & $1.9 \mathrm{e}-08$ & $1.1 \mathrm{e}-08$ & $8.2 \mathrm{e}-07$ & $5.9 \mathrm{e}-05$ & $9.0 \mathrm{e}-09$ \\
\hline & 1.5 & 0.8 & $2.0 \mathrm{e}-09$ & $7.9 \mathrm{e}-11$ & $5.5 \mathrm{e}-08$ & $1.2 \mathrm{e}-08$ & $4.5 \mathrm{e}-09$ & $3.8 \mathrm{e}-09$ & $1.4 \mathrm{e}-06$ & $1.7 \mathrm{e}-06$ & $3.3 \mathrm{e}-09$ \\
\hline & 1.5 & 1.2 & $4.1 \mathrm{e}-09$ & $6.4 \mathrm{e}-10$ & $3.2 \mathrm{e}-08$ & 7.0e-08 & $6.8 \mathrm{e}-09$ & $6.3 e-09$ & $3.7 \mathrm{e}-07$ & $2.6 \mathrm{e}-05$ & $1.2 \mathrm{e}-08$ \\
\hline \multirow[t]{8}{*}{50} & 0.5 & 0.8 & $1.0 \mathrm{e}-08$ & $3.8 \mathrm{e}-10$ & $5.1 \mathrm{e}-08$ & $2.1 \mathrm{e}-08$ & $8.7 \mathrm{e}-09$ & $1.6 \mathrm{e}-10$ & $1.1 \mathrm{e}-06$ & $1.7 \mathrm{e}-06$ & $3.2 \mathrm{e}-09$ \\
\hline & 0.5 & 1.2 & $9.3 \mathrm{e}-08$ & $1.6 \mathrm{e}-09$ & $9.0 \mathrm{e}-08$ & $3.3 \mathrm{e}-07$ & $4.9 \mathrm{e}-08$ & $9.9 \mathrm{e}-10$ & $8.0 \mathrm{e}-07$ & $6.6 \mathrm{e}-05$ & $9.0 \mathrm{e}-08$ \\
\hline & 0.5 & 0.8 & $1.1 \mathrm{e}-08$ & $3.4 \mathrm{e}-10$ & $6.2 \mathrm{e}-08$ & $2.4 \mathrm{e}-08$ & $1.0 \mathrm{e}-08$ & $3.7 \mathrm{e}-10$ & $1.3 \mathrm{e}-06$ & $2.1 \mathrm{e}-06$ & $2.5 \mathrm{e}-09$ \\
\hline & 0.5 & 1.2 & $1.1 \mathrm{e}-07$ & $3.0 \mathrm{e}-09$ & $9.6 \mathrm{e}-08$ & $3.8 \mathrm{e}-07$ & $5.7 \mathrm{e}-08$ & $2.9 \mathrm{e}-10$ & $8.2 \mathrm{e}-07$ & $6.9 \mathrm{e}-05$ & $1.4 \mathrm{e}-07$ \\
\hline & 1.5 & 0.8 & $6.0 \mathrm{e}-09$ & $3.6 \mathrm{e}-12$ & $7.5 \mathrm{e}-08$ & $2.1 \mathrm{e}-08$ & $8.2 \mathrm{e}-09$ & $2.6 \mathrm{e}-09$ & $1.8 \mathrm{e}-06$ & $2.4 \mathrm{e}-06$ & $3.3 \mathrm{e}-10$ \\
\hline & 1.5 & 1.2 & $2.3 \mathrm{e}-09$ & $2.0 \mathrm{e}-10$ & $1.4 \mathrm{e}-08$ & $3.2 \mathrm{e}-08$ & $3.2 \mathrm{e}-09$ & $2.4 \mathrm{e}-09$ & $1.5 \mathrm{e}-07$ & $1.1 \mathrm{e}-05$ & $3.3 \mathrm{e}-09$ \\
\hline & 1.5 & 0.8 & $1.7 \mathrm{e}-09$ & $5.6 \mathrm{e}-12$ & $3.0 \mathrm{e}-08$ & $1.0 \mathrm{e}-08$ & $4.0 \mathrm{e}-09$ & $2.3 e-09$ & $1.1 \mathrm{e}-06$ & $1.4 \mathrm{e}-06$ & $1.2 \mathrm{e}-09$ \\
\hline & 1.5 & 1.2 & $5.1 \mathrm{e}-09$ & $3.5 \mathrm{e}-10$ & $2.7 \mathrm{e}-08$ & $6.5 \mathrm{e}-08$ & $6.7 \mathrm{e}-09$ & $4.6 \mathrm{e}-09$ & $3.1 \mathrm{e}-07$ & $2.2 \mathrm{e}-05$ & $5.5 \mathrm{e}-09$ \\
\hline \multirow[t]{8}{*}{100} & 0.5 & 0.8 & $1.0 \mathrm{e}-08$ & $6.1 \mathrm{e}-10$ & $3.4 \mathrm{e}-08$ & $1.7 \mathrm{e}-08$ & $7.2 \mathrm{e}-09$ & $1.9 \mathrm{e}-11$ & $6.7 \mathrm{e}-07$ & $1.2 \mathrm{e}-06$ & $6.6 \mathrm{e}-09$ \\
\hline & 0.5 & 1.2 & $2.6 \mathrm{e}-08$ & $5.5 \mathrm{e}-10$ & $2.4 \mathrm{e}-08$ & $9.1 \mathrm{e}-08$ & $1.3 \mathrm{e}-08$ & $1.7 \mathrm{e}-10$ & $2.1 \mathrm{e}-07$ & $1.7 \mathrm{e}-05$ & $2.8 \mathrm{e}-08$ \\
\hline & 0.5 & 0.8 & $9.0 \mathrm{e}-09$ & $4.9 \mathrm{e}-10$ & $3.3 \mathrm{e}-08$ & $1.5 \mathrm{e}-08$ & $6.6 \mathrm{e}-09$ & $6.1 \mathrm{e}-13$ & $6.6 \mathrm{e}-07$ & $1.1 \mathrm{e}-06$ & $5.1 \mathrm{e}-09$ \\
\hline & 0.5 & 1.2 & $3.0 \mathrm{e}-08$ & $7.3 \mathrm{e}-10$ & $2.5 \mathrm{e}-08$ & $9.8 \mathrm{e}-08$ & $1.4 \mathrm{e}-08$ & $9.7 \mathrm{e}-11$ & $2.1 \mathrm{e}-07$ & $1.8 \mathrm{e}-05$ & $3.6 \mathrm{e}-08$ \\
\hline & 1.5 & 0.8 & $5.2 \mathrm{e}-10$ & $3.1 \mathrm{e}-12$ & $9.7 \mathrm{e}-09$ & $2.4 \mathrm{e}-09$ & $9.1 \mathrm{e}-10$ & $5.1 \mathrm{e}-10$ & $2.4 \mathrm{e}-07$ & $3.1 \mathrm{e}-07$ & $2.6 \mathrm{e}-10$ \\
\hline & 1.5 & 1.2 & $1.2 \mathrm{e}-09$ & $5.7 \mathrm{e}-11$ & $6.0 \mathrm{e}-09$ & $1.4 \mathrm{e}-08$ & $1.5 \mathrm{e}-09$ & $9.1 \mathrm{e}-10$ & $6.6 \mathrm{e}-08$ & $4.8 \mathrm{e}-06$ & $7.8 \mathrm{e}-10$ \\
\hline & 1.5 & 0.8 & $8.6 \mathrm{e}-10$ & $2.7 \mathrm{e}-13$ & $1.2 \mathrm{e}-08$ & $3.4 \mathrm{e}-09$ & $1.3 e-09$ & $5.5 \mathrm{e}-10$ & $3.1 \mathrm{e}-07$ & $4.1 \mathrm{e}-07$ & $1.6 \mathrm{e}-10$ \\
\hline & 1.5 & 1.2 & $1.0 \mathrm{e}-09$ & $7.5 \mathrm{e}-11$ & $5.7 \mathrm{e}-09$ & $1.3 \mathrm{e}-08$ & $1.3 e-09$ & $9.6 \mathrm{e}-10$ & $6.3 \mathrm{e}-08$ & $4.5 \mathrm{e}-06$ & $1.2 \mathrm{e}-09$ \\
\hline
\end{tabular}

From Tables 4.3 and 4.4, Bayesian estimation under LINEX loss gives smaller Mean Squared Error as compared to the others. It is observed again from Table 3.4 that as the sample size increases, the Mean Squared Error values of the MLE has increased and Bayes estimation under all loss functions have decreases.

In Table 4.5 we present the Absolute Bias estimated values for the scale parameter $\sigma$ for both the Maximum Likelihood Estimation and Bayesian Estimation using extension of Jeffrey's prior information with the three loss functions.

Table 4.5:- Absolute Bias Estimated Parameter $(\sigma)$ using Extension of Jeffrey's prior information

\begin{tabular}{|c|c|c|c|c|c|c|c|c|c|c|c|c|c|}
\hline \multirow[b]{2}{*}{$n$} & \multirow[b]{2}{*}{$\sigma$} & \multirow[b]{2}{*}{$c$} & \multirow[b]{2}{*}{$\beta$} & \multirow[b]{2}{*}{$\hat{\sigma}_{M L}$} & \multirow[b]{2}{*}{$\hat{\sigma}_{B S}$} & $\hat{\sigma}_{B L}$ & $\hat{\sigma}_{B G}$ & $\hat{\sigma}_{B L}$ & $\hat{\sigma}_{B G}$ & $\hat{\sigma}_{B L}$ & $\hat{\sigma}_{B G}$ & $\hat{\sigma}_{B L}$ & $\hat{\sigma}_{B G}$ \\
\hline & & & & & & \multicolumn{2}{|c|}{$\mathrm{a}=\mathrm{k}=0.6$} & \multicolumn{2}{|c|}{$\mathrm{a}=\mathrm{k}=-0.6$} & \multicolumn{2}{|c|}{$\mathrm{a}=\mathrm{k}=1.6$} & \multicolumn{2}{|c|}{$\mathrm{a}=\mathrm{k}=-1.6$} \\
\hline \multirow[t]{8}{*}{25} & 0.5 & 0.4 & 0.8 & 0.0138 & $1.5 \mathrm{e}-05$ & $7.7 \mathrm{e}-06$ & $5.7 \mathrm{e}-06$ & $1.0 \mathrm{e}-05$ & $9.6 \mathrm{e}-06$ & $1.5 \mathrm{e}-05$ & $4.2 \mathrm{e}-05$ & $3.2 \mathrm{e}-05$ & $2.0 \mathrm{e}-05$ \\
\hline & 0.5 & 0.4 & 1.2 & 0.0136 & $1.6 \mathrm{e}-05$ & $8.2 \mathrm{e}-06$ & $6.5 \mathrm{e}-06$ & $1.1 \mathrm{e}-05$ & $1.0 \mathrm{e}-05$ & $1.6 \mathrm{e}-05$ & $4.2 \mathrm{e}-05$ & $3.5 \mathrm{e}-05$ & $2.2 \mathrm{e}-05$ \\
\hline & 0.5 & 1.4 & 0.8 & 0.0164 & $1.9 \mathrm{e}-05$ & $7.9 \mathrm{e}-06$ & $5.2 \mathrm{e}-05$ & $1.7 \mathrm{e}-05$ & $1.7 \mathrm{e}-05$ & $1.0 \mathrm{e}-05$ & 0.00033 & $8.5 \mathrm{e}-05$ & $1.6 \mathrm{e}-05$ \\
\hline & 0.5 & 1.4 & 1.2 & 0.0108 & $3.3 e-05$ & $1.3 \mathrm{e}-05$ & $9.6 e-05$ & $3.0 \mathrm{e}-05$ & $3.0 \mathrm{e}-05$ & $1.7 \mathrm{e}-05$ & 0.00063 & 0.00015 & $2.7 e-05$ \\
\hline & 1.5 & 0.4 & 0.8 & 0.0209 & 0.00120 & 0.00033 & 0.00028 & 0.00154 & 0.00057 & 0.00023 & 0.00041 & 0.01371 & 0.00267 \\
\hline & 1.5 & 0.4 & 1.2 & 0.0349 & $6.3 e-05$ & $2.2 \mathrm{e}-05$ & $3.3 e-06$ & $4.9 \mathrm{e}-05$ & $2.5 \mathrm{e}-05$ & $2.0 \mathrm{e}-05$ & $1.2 \mathrm{e}-05$ & 0.00027 & 0.00016 \\
\hline & 1.5 & 1.4 & 0.8 & 0.0504 & $4.3 e-05$ & $1.4 \mathrm{e}-05$ & $5.2 \mathrm{e}-06$ & $4.1 \mathrm{e}-05$ & $1.8 \mathrm{e}-05$ & $1.2 \mathrm{e}-05$ & $4.7 \mathrm{e}-08$ & $5.7 \mathrm{e}-05$ & 0.00010 \\
\hline & 1.5 & 1.4 & 1.2 & 0.0001 & $4.7 \mathrm{e}-05$ & $1.5 \mathrm{e}-05$ & $5.7 \mathrm{e}-06$ & $4.5 \mathrm{e}-05$ & $2.0 \mathrm{e}-05$ & $1.3 \mathrm{e}-05$ & $3.2 \mathrm{e}-07$ & $7.3 e-05$ & 0.00011 \\
\hline \multirow[t]{8}{*}{50} & 0.5 & 0.4 & 0.8 & 0.0371 & $4.2 \mathrm{e}-06$ & $2.2 \mathrm{e}-06$ & $6.6 \mathrm{e}-07$ & $2.8 \mathrm{e}-06$ & $2.6 \mathrm{e}-06$ & $4.4 \mathrm{e}-06$ & $2.0 \mathrm{e}-05$ & $8.2 \mathrm{e}-06$ & $6.0 \mathrm{e}-06$ \\
\hline & 0.5 & 0.4 & 1.2 & 0.0237 & $1.2 \mathrm{e}-05$ & $6.2 \mathrm{e}-06$ & 7.1e-06 & $8.8 \mathrm{e}-06$ & $8.2 \mathrm{e}-06$ & $1.2 \mathrm{e}-05$ & $1.38 \mathrm{e}-05$ & $2.9 \mathrm{e}-05$ & $1.6 \mathrm{e}-05$ \\
\hline & 0.5 & 1.4 & 0.8 & 0.0410 & 7.4e-06 & $3.0 \mathrm{e}-06$ & $1.9 \mathrm{e}-05$ & $6.5 \mathrm{e}-06$ & $6.5 \mathrm{e}-06$ & $4.1 \mathrm{e}-06$ & 0.00012 & $3.2 \mathrm{e}-05$ & $6.5 \mathrm{e}-06$ \\
\hline & 0.5 & 1.4 & 1.2 & 0.0190 & $1.9 \mathrm{e}-05$ & $7.4 \mathrm{e}-06$ & $6.2 \mathrm{e}-05$ & $1.8 \mathrm{e}-05$ & $1.8 \mathrm{e}-05$ & $8.7 \mathrm{e}-06$ & 0.00042 & $9.6 \mathrm{e}-05$ & $1.4 \mathrm{e}-05$ \\
\hline & 1.5 & 0.4 & 0.8 & 0.0563 & 7.4e-05 & $2.4 \mathrm{e}-05$ & $9.3 e-06$ & 7.2e-05 & $3.2 \mathrm{e}-05$ & $2.0 \mathrm{e}-05$ & $1.18 \mathrm{e}-06$ & 0.00013 & 0.00018 \\
\hline & 1.5 & 0.4 & 1.2 & 0.0882 & $1.6 \mathrm{e}-05$ & $6.5 \mathrm{e}-06$ & $1.5 \mathrm{e}-07$ & $1.0 \mathrm{e}-05$ & $6.4 \mathrm{e}-06$ & $6.2 \mathrm{e}-06$ & $6.32 \mathrm{e}-06$ & 0.00016 & $4.7 \mathrm{e}-05$ \\
\hline & 1.5 & 1.4 & 0.8 & 0.0621 & $7.5 e-05$ & $1.4 \mathrm{e}-05$ & 7.0e-06 & $8.2 \mathrm{e}-05$ & $3.3 e-05$ & $1.8 \mathrm{e}-05$ & $1.05 \mathrm{e}-05$ & 0.00040 & 0.00017 \\
\hline & 1.5 & 1.4 & 1.2 & 0.0607 & $8.2 \mathrm{e}-05$ & $2.5 \mathrm{e}-05$ & $1.4 \mathrm{e}-05$ & $9.0 \mathrm{e}-05$ & $3.7 \mathrm{e}-05$ & $1.9 \mathrm{e}-05$ & $1.24 \mathrm{e}-05$ & 0.00047 & 0.00019 \\
\hline \multirow[t]{8}{*}{100} & 0.5 & 0.4 & 0.8 & 0.0571 & $3.8 \mathrm{e}-06$ & $1.9 \mathrm{e}-06$ & $1.4 \mathrm{e}-06$ & $2.6 \mathrm{e}-06$ & $2.4 \mathrm{e}-06$ & $3.8 \mathrm{e}-06$ & $1.0 \mathrm{e}-05$ & $8.3 e-06$ & $5.3 e-06$ \\
\hline & 0.5 & 0.4 & 1.2 & 0.0453 & $7.5 e-06$ & $3.7 \mathrm{e}-06$ & $4.8 \mathrm{e}-06$ & $5.3 e-06$ & $5.0 \mathrm{e}-06$ & 7.1e-06 & $3.62 \mathrm{e}-06$ & $1.8 \mathrm{e}-05$ & $9.8 \mathrm{e}-06$ \\
\hline & 0.5 & 1.4 & 0.8 & 0.0567 & $6.2 \mathrm{e}-06$ & $2.4 \mathrm{e}-06$ & $1.6 \mathrm{e}-05$ & $5.4 \mathrm{e}-06$ & $5.5 \mathrm{e}-06$ & $3.3 \mathrm{e}-06$ & 0.0001 & $2.7 \mathrm{e}-05$ & $5.3 e-06$ \\
\hline & 0.5 & 1.4 & 1.2 & 0.0350 & $9.4 \mathrm{e}-06$ & $3.3 \mathrm{e}-06$ & $3.4 \mathrm{e}-05$ & $9.0 \mathrm{e}-06$ & $9.3 e-06$ & $3.3 e-06$ & 0.00024 & $5.0 \mathrm{e}-05$ & $6.1 \mathrm{e}-06$ \\
\hline & 1.5 & 0.4 & 0.8 & 0.1354 & $1.8 \mathrm{e}-05$ & $6.5 \mathrm{e}-06$ & $1.2 \mathrm{e}-06$ & $1.5 \mathrm{e}-05$ & $7.6 \mathrm{e}-06$ & $5.9 \mathrm{e}-06$ & $2.80 \mathrm{e}-06$ & $5.6 \mathrm{e}-05$ & $4.9 \mathrm{e}-05$ \\
\hline & 1.5 & 0.4 & 1.2 & 0.1428 & $1.5 \mathrm{e}-05$ & $5.6 \mathrm{e}-06$ & $8.3 e-07$ & $1.2 \mathrm{e}-05$ & $6.3 \mathrm{e}-06$ & $5.1 \mathrm{e}-06$ & $3.08 \mathrm{e}-06$ & $6.8 \mathrm{e}-05$ & $4.2 \mathrm{e}-05$ \\
\hline & 1.5 & 1.4 & 0.8 & 0.1381 & $2.8 \mathrm{e}-05$ & $8.7 \mathrm{e}-06$ & $4.4 \mathrm{e}-06$ & $2.9 \mathrm{e}-05$ & $1.2 \mathrm{e}-05$ & $7.1 \mathrm{e}-06$ & $2.95 \mathrm{e}-06$ & 0.00012 & $6.8 \mathrm{e}-05$ \\
\hline & 1.5 & 1.4 & 1.2 & 0.1439 & $2.5 \mathrm{e}-05$ & $7.9 \mathrm{e}-06$ & $3.8 \mathrm{e}-06$ & $2.6 \mathrm{e}-05$ & $1.1 \mathrm{e}-05$ & $6.4 \mathrm{e}-06$ & $2.31 \mathrm{e}-06$ & 0.00010 & $6.1 \mathrm{e}-05$ \\
\hline
\end{tabular}


ML: Maximum Likelihood, BS: Squared Error Loss function, BL: LINEX Loss function, BG: General Entropy Loss function

In Table 4.6 we present the Absolute Bias estimated values for the scale parameter $\sigma$ for the MLE and Bayesian Estimation using Jeffreys prior information with the three loss functions.

Table 4.6:- Absolute Bias Estimated Parameter $(\boldsymbol{\sigma})$ using Jeffreys prior information

\begin{tabular}{|c|c|c|c|c|c|c|c|c|c|c|c|}
\hline \multirow[b]{2}{*}{$n$} & \multirow[b]{2}{*}{$\sigma$} & \multirow[b]{2}{*}{$\beta$} & \multirow[b]{2}{*}{$\hat{\sigma}_{B S}$} & $\hat{\sigma}_{B L}$ & $\hat{\sigma}_{B G}$ & $\hat{\sigma}_{B L}$ & $\hat{\sigma}_{B G}$ & $\hat{\sigma}_{B L}$ & $\hat{\sigma}_{B G}$ & $\hat{\sigma}_{B L}$ & $\hat{\sigma}_{B G}$ \\
\hline & & & & \multicolumn{2}{|c|}{$\mathrm{a}=\mathrm{k}=0.6$} & \multicolumn{2}{|c|}{$a=k=-0.6$} & \multicolumn{2}{|c|}{$\mathrm{a}=\mathrm{k}=1.6$} & \multicolumn{2}{|c|}{$a=k=-1.6$} \\
\hline \multirow[t]{8}{*}{25} & 0.5 & 0.8 & $1.3 e-05$ & $6.9 \mathrm{e}-06$ & $2.1 \mathrm{e}-06$ & $8.9 \mathrm{e}-06$ & $8.1 \mathrm{e}-06$ & $1.3 e-05$ & $6.1 \mathrm{e}-05$ & $2.5 \mathrm{e}-05$ & $1.8 \mathrm{e}-05$ \\
\hline & 0.5 & 1.2 & $1.4 \mathrm{e}-05$ & $7.3 \mathrm{e}-06$ & $2.7 \mathrm{e}-06$ & $9.6 \mathrm{e}-06$ & $8.7 \mathrm{e}-06$ & $1.4 \mathrm{e}-05$ & $6.2 \mathrm{e}-05$ & $2.8 \mathrm{e}-05$ & $2.0 \mathrm{e}-05$ \\
\hline & 0.5 & 0.8 & $9.0 \mathrm{e}-06$ & $4.7 \mathrm{e}-06$ & $2.5 \mathrm{e}-07$ & $5.9 \mathrm{e}-06$ & $5.3 e-06$ & $9.8 \mathrm{e}-06$ & $5.7 \mathrm{e}-05$ & $1.5 \mathrm{e}-05$ & $1.3 \mathrm{e}-05$ \\
\hline & 0.5 & 1.2 & $2.7 \mathrm{e}-05$ & $1.3 e-05$ & $1.4 \mathrm{e}-05$ & $1.9 \mathrm{e}-05$ & $1.7 \mathrm{e}-05$ & $2.6 \mathrm{e}-05$ & $4.4 \mathrm{e}-05$ & $6.2 \mathrm{e}-05$ & $3.6 \mathrm{e}-05$ \\
\hline & 1.5 & 0.8 & 0.00123 & 0.00034 & 0.00029 & 0.00158 & 0.00059 & 0.00024 & 0.00043 & 0.01424 & 0.00273 \\
\hline & 1.5 & 1.2 & $6.8 \mathrm{e}-05$ & $2.3 e-05$ & $5.0 \mathrm{e}-06$ & $5.7 \mathrm{e}-05$ & $2.8 \mathrm{e}-05$ & $2.1 \mathrm{e}-05$ & $9.2 \mathrm{e}-06$ & 0.00017 & 0.00018 \\
\hline & 1.5 & 0.8 & $2.6 \mathrm{e}-05$ & $1.0 \mathrm{e}-05$ & $2.1 \mathrm{e}-07$ & $1.6 e-05$ & $9.9 \mathrm{e}-06$ & $9.6 \mathrm{e}-06$ & $9.7 \mathrm{e}-06$ & 0.00024 & $7.3 e-05$ \\
\hline & 1.5 & 1.2 & $2.8 \mathrm{e}-05$ & $1.0 \mathrm{e}-05$ & $1.9 \mathrm{e}-08$ & $1.8 \mathrm{e}-05$ & $1.0 \mathrm{e}-05$ & $1.0 \mathrm{e}-05$ & $1.0 \mathrm{e}-05$ & 0.00025 & $8.0 \mathrm{e}-05$ \\
\hline \multirow[t]{8}{*}{50} & 0.5 & 0.8 & $3.6 \mathrm{e}-06$ & $1.9 \mathrm{e}-06$ & $4.9 \mathrm{e}-07$ & $2.3 e-06$ & $2.1 \mathrm{e}-06$ & $4.0 \mathrm{e}-06$ & $2.6 \mathrm{e}-05$ & $6.0 \mathrm{e}-06$ & $5.3 e-06$ \\
\hline & 0.5 & 1.2 & $1.2 \mathrm{e}-07$ & $5.3 e-06$ & $2.1 \mathrm{e}-08$ & $7.7 \mathrm{e}-06$ & $7.1 \mathrm{e}-06$ & $1.1 \mathrm{e}-05$ & $2.7 \mathrm{e}-05$ & $2.4 \mathrm{e}-05$ & $1.5 \mathrm{e}-05$ \\
\hline & 0.5 & 0.8 & $3.0 \mathrm{e}-06$ & $1.6 \mathrm{e}-06$ & $6.8 \mathrm{e}-07$ & $1.9 \mathrm{e}-06$ & $1.7 \mathrm{e}-06$ & $3.3 e-06$ & $2.4 \mathrm{e}-05$ & $4.6 \mathrm{e}-06$ & $4.5 \mathrm{e}-06$ \\
\hline & 0.5 & 1.2 & $2.6 \mathrm{e}-05$ & $1.3 e-05$ & $2.1 \mathrm{e}-05$ & $1.9 \mathrm{e}-05$ & $1.8 \mathrm{e}-05$ & $2.4 \mathrm{e}-05$ & $2.4 \mathrm{e}-05$ & $6.8 \mathrm{e}-05$ & $3.4 \mathrm{e}-05$ \\
\hline & 1.5 & 0.8 & $7.8 \mathrm{e}-05$ & $2.5 \mathrm{e}-05$ & $1.0 \mathrm{e}-05$ & $7.9 \mathrm{e}-05$ & $3.4 \mathrm{e}-05$ & $2.1 \mathrm{e}-05$ & $3.7 \mathrm{e}-06$ & 0.00021 & 0.00019 \\
\hline & 1.5 & 1.2 & $1.8 \mathrm{e}-05$ & $6.9 \mathrm{e}-06$ & $4.2 \mathrm{e}-07$ & $1.3 \mathrm{e}-05$ & $7.3 \mathrm{e}-06$ & $6.5 e-06$ & $5.3 e-06$ & 0.00012 & $5.1 \mathrm{e}-05$ \\
\hline & 1.5 & 0.8 & $3.0 \mathrm{e}-05$ & $1.0 \mathrm{e}-05$ & $2.0 \mathrm{e}-06$ & $4.9 \mathrm{e}-05$ & $2.2 \mathrm{e}-05$ & $1.5 \mathrm{e}-05$ & $1.9 \mathrm{e}-06$ & $1.5 \mathrm{e}-05$ & 0.00013 \\
\hline & 1.5 & 1.2 & $5.8 \mathrm{e}-05$ & $1.9 \mathrm{e}-05$ & $6.7 \mathrm{e}-06$ & $5.5 e-05$ & $2.5 \mathrm{e}-05$ & $1.6 \mathrm{e}-05$ & $8.6 e-07$ & $5.3 e-05$ & 0.00014 \\
\hline \multirow[t]{8}{*}{100} & 0.5 & 0.8 & $3.3 e-06$ & $1.7 \mathrm{e}-06$ & $5.7 \mathrm{e}-07$ & $2.2 \mathrm{e}-06$ & $2.0 \mathrm{e}-06$ & $3.5 \mathrm{e}-06$ & $1.5 e-05$ & $6.5 \mathrm{e}-06$ & $4.7 \mathrm{e}-06$ \\
\hline & 0.5 & 1.2 & $6.7 \mathrm{e}-06$ & $3.4 \mathrm{e}-06$ & $3.4 \mathrm{e}-06$ & $4.7 \mathrm{e}-06$ & $4.3 \mathrm{e}-06$ & $6.6 \mathrm{e}-06$ & $1.1 \mathrm{e}-05$ & $1.5 \mathrm{e}-05$ & $9.0 \mathrm{e}-06$ \\
\hline & 0.5 & 0.8 & $3.4 \mathrm{e}-06$ & $1.7 \mathrm{e}-06$ & $6.3 e-07$ & $2.3 \mathrm{e}-06$ & $2.1 \mathrm{e}-06$ & $3.6 \mathrm{e}-06$ & $1.5 \mathrm{e}-05$ & $6.7 \mathrm{e}-06$ & $4.8 \mathrm{e}-06$ \\
\hline & 0.5 & 1.2 & $2.0 \mathrm{e}-05$ & $9.7 \mathrm{e}-06$ & $1.9 \mathrm{e}-05$ & $1.4 \mathrm{e}-05$ & $1.4 \mathrm{e}-05$ & $1.7 \mathrm{e}-05$ & $4.5 e-05$ & 0.00013 & $6.0 \mathrm{e}-06$ \\
\hline & 1.5 & 0.8 & $2.0 \mathrm{e}-05$ & $6.9 \mathrm{e}-06$ & $1.7 \mathrm{e}-06$ & $1.7 \mathrm{e}-05$ & $8.4 \mathrm{e}-06$ & $6.1 \mathrm{e}-06$ & $1.9 \mathrm{e}-06$ & $2.9 \mathrm{e}-05$ & $5.2 \mathrm{e}-05$ \\
\hline & 1.5 & 1.2 & $1.7 \mathrm{e}-05$ & $5.9 \mathrm{e}-06$ & $1.2 \mathrm{e}-06$ & $1.4 \mathrm{e}-05$ & $7.0 \mathrm{e}-06$ & $5.3 e-06$ & $2.3 e-06$ & $4.4 \mathrm{e}-05$ & $4.5 e-05$ \\
\hline & 1.5 & 0.8 & $1.9 \mathrm{e}-05$ & $6.5 e-06$ & $1.5 \mathrm{e}-06$ & $1.6 \mathrm{e}-05$ & $7.9 \mathrm{e}-06$ & $5.8 \mathrm{e}-06$ & $2.1 \mathrm{e}-06$ & $3.5 e-05$ & $4.9 \mathrm{e}-05$ \\
\hline & 1.5 & 1.2 & $1.6 \mathrm{e}-05$ & $5.8 \mathrm{e}-06$ & $1.2 \mathrm{e}-06$ & $1.4 \mathrm{e}-05$ & $6.9 \mathrm{e}-06$ & $5.2 \mathrm{e}-06$ & $2.3 e-06$ & $4.5 e-05$ & $4.4 \mathrm{e}-05$ \\
\hline
\end{tabular}

From Table 4.5 and 4.6 it is observed that Bayes estimation with LINEX loss function provides the smallest Absolute Bias values in most cases and as the sample size increases Absolute values of the MLE increased and Bayes estimation under all loss functions decreases.

In Table 4.7 we present the Absolute Bias estimated values for the shape parameter $\boldsymbol{\beta}$ for both the Maximum Likelihood Estimation and Bayesian Estimation using extension of Jeffrey's prior information with the three loss functions.

Table 4.7:- Absolute Bias Estimated Parameter $(\boldsymbol{\beta})$ using extension of Jeffrey’s prior information

\begin{tabular}{|c|c|c|c|c|c|c|c|c|c|c|c|c|c|}
\hline \multirow[b]{2}{*}{$\mathrm{n}$} & \multirow[b]{2}{*}{$\sigma$} & \multirow[b]{2}{*}{$\mathrm{c}$} & \multirow[b]{2}{*}{$\beta$} & \multirow[b]{2}{*}{$\hat{\beta}_{M L}$} & \multirow[b]{2}{*}{$\hat{\beta}_{B S}$} & $\hat{\beta}_{B L}$ & $\hat{\beta}_{B G}$ & $\hat{\beta}_{B L}$ & $\hat{\beta}_{B G}$ & $\hat{\beta}_{B L}$ & $\hat{\beta}_{B G}$ & $\hat{\beta}_{B L}$ & $\hat{\beta}_{B G}$ \\
\hline & & & & & & \multicolumn{2}{|c|}{$\mathrm{a}=\mathrm{k}=0.6$} & \multicolumn{2}{|c|}{$\mathrm{a}=\mathrm{k}=-0.6$} & \multicolumn{2}{|c|}{$\mathrm{a}=\mathrm{k}=1.6$} & \multicolumn{2}{|c|}{$\mathrm{a}=\mathrm{k}=-1.6$} \\
\hline \multirow[t]{8}{*}{25} & 0.5 & 0.4 & 0.8 & 0.0010 & $1.1 \mathrm{e}-05$ & $2.7 \mathrm{e}-06$ & $2.1 \mathrm{e}-05$ & $1.5 \mathrm{e}-05$ & $9.8 \mathrm{e}-06$ & $2.6 \mathrm{e}-07$ & $9.7 e-05$ & 0.00013 & $9.0 \mathrm{e}-06$ \\
\hline & 0.5 & 0.4 & 1.2 & 0.0014 & $1.9 \mathrm{e}-05$ & $2.9 \mathrm{e}-06$ & $1.8 \mathrm{e}-05$ & $3.6 e-05$ & $1.4 \mathrm{e}-05$ & $1.5 \mathrm{e}-06$ & $5.5 \mathrm{e}-05$ & 0.00050 & $2.0 \mathrm{e}-05$ \\
\hline & 0.5 & 1.4 & 0.8 & 0.0009 & $1.7 \mathrm{e}-05$ & $5.0 \mathrm{e}-06$ & $2.6 \mathrm{e}-05$ & $2.0 \mathrm{e}-05$ & $1.3 \mathrm{e}-05$ & $3.1 \mathrm{e}-06$ & 0.0001 & 0.00016 & $1.7 \mathrm{e}-05$ \\
\hline & 0.5 & 1.4 & 1.2 & 0.0022 & $1.3 e-05$ & $6.5 \mathrm{e}-07$ & $1.6 \mathrm{e}-05$ & $2.9 \mathrm{e}-05$ & $1.0 \mathrm{e}-05$ & $3.7 \mathrm{e}-06$ & $5.2 \mathrm{e}-05$ & 0.00046 & $7.2 \mathrm{e}-06$ \\
\hline & 1.5 & 0.4 & 0.8 & 0.0008 & $4.7 \mathrm{e}-06$ & $5.3 e-08$ & $1.8 \mathrm{e}-05$ & $9.3 e-06$ & $5.8 \mathrm{e}-06$ & $3.7 \mathrm{e}-06$ & $8.9 \mathrm{e}-05$ & 0.00010 & $1.9 \mathrm{e}-06$ \\
\hline & 1.5 & 0.4 & 1.2 & 0.0007 & $4.7 \mathrm{e}-06$ & $6.1 \mathrm{e}-07$ & $8.9 \mathrm{e}-06$ & $1.4 \mathrm{e}-05$ & $4.7 \mathrm{e}-06$ & $3.1 \mathrm{e}-06$ & $2.9 \mathrm{e}-05$ & 0.00025 & $1.7 \mathrm{e}-06$ \\
\hline & 1.5 & 1.4 & 0.8 & 0.0003 & $5.9 \mathrm{e}-06$ & $1.3 \mathrm{e}-06$ & $1.1 \mathrm{e}-05$ & $7.8 \mathrm{e}-06$ & $5.0 \mathrm{e}-06$ & $4.4 \mathrm{e}-08$ & $5.0 \mathrm{e}-05$ & $6.7 \mathrm{e}-05$ & $4.4 \mathrm{e}-06$ \\
\hline & 1.5 & 1.4 & 1.2 & 0.0006 & $4.0 \mathrm{e}-06$ & $1.8 \mathrm{e}-06$ & $4.0 \mathrm{e}-06$ & $3.8 \mathrm{e}-06$ & $5.4 \mathrm{e}-07$ & $3.3 \mathrm{e}-06$ & $1.5 \mathrm{e}-05$ & 0.00012 & $1.0 \mathrm{e}-05$ \\
\hline \multirow[t]{5}{*}{50} & 0.5 & 0.4 & 0.8 & 0.0008 & $2.9 \mathrm{e}-06$ & $5.1 \mathrm{e}-07$ & $6.9 \mathrm{e}-06$ & $4.3 \mathrm{e}-06$ & $2.7 \mathrm{e}-06$ & $5.3 e-07$ & $3.2 \mathrm{e}-05$ & $4.0 \mathrm{e}-05$ & $1.4 \mathrm{e}-06$ \\
\hline & 0.5 & 0.4 & 1.2 & 0.0015 & $1.0 \mathrm{e}-05$ & $1.4 \mathrm{e}-06$ & $9.7 \mathrm{e}-06$ & $1.9 \mathrm{e}-05$ & $7.3 \mathrm{e}-06$ & $8.7 \mathrm{e}-07$ & $2.8 \mathrm{e}-05$ & 0.00026 & $1.0 \mathrm{e}-05$ \\
\hline & 0.5 & 1.4 & 0.8 & 0.0010 & 7.0e-06 & $1.9 \mathrm{e}-06$ & $1.1 \mathrm{e}-05$ & $8.5 \mathrm{e}-06$ & $5.5 \mathrm{e}-06$ & $1.0 \mathrm{e}-06$ & $4.7 \mathrm{e}-05$ & $6.7 \mathrm{e}-05$ & $6.6 \mathrm{e}-06$ \\
\hline & 0.5 & 1.4 & 1.2 & 0.0011 & 7.0e-06 & $6.1 \mathrm{e}-07$ & 8.0e-06 & $1.4 \mathrm{e}-05$ & $5.4 \mathrm{e}-06$ & $1.4 \mathrm{e}-06$ & $2.4 \mathrm{e}-05$ & 0.00022 & $5.2 \mathrm{e}-06$ \\
\hline & 1.5 & 0.4 & 0.8 & 0.0008 & $2.0 \mathrm{e}-06$ & $9.4 \mathrm{e}-08$ & $8.3 \mathrm{e}-06$ & $4.2 \mathrm{e}-06$ & $2.5 \mathrm{e}-06$ & $1.8 \mathrm{e}-06$ & $4.1 \mathrm{e}-05$ & $4.7 \mathrm{e}-05$ & $1.1 \mathrm{e}-06$ \\
\hline
\end{tabular}




\begin{tabular}{|l|l|l|l|l|l|l|l|l|l|l|l|l|l|}
\hline & 1.5 & 0.4 & 1.2 & 0.0007 & $1.8 \mathrm{e}-06$ & $3.5 \mathrm{e}-07$ & $3.8 \mathrm{e}-06$ & $6.0 \mathrm{e}-06$ & $1.9 \mathrm{e}-06$ & $1.4 \mathrm{e}-06$ & $1.2 \mathrm{e}-05$ & 0.00010 & $1.2 \mathrm{e}-06$ \\
& 1.5 & 1.4 & 0.8 & 0.0006 & $5.3 \mathrm{e}-06$ & $1.1 \mathrm{e}-06$ & $8.2 \mathrm{e}-06$ & $7.0 \mathrm{e}-06$ & $4.5 \mathrm{e}-06$ & $1.7 \mathrm{e}-07$ & $1.7 \mathrm{e}-07$ & $5.9 \mathrm{e}-05$ & $4.2 \mathrm{e}-06$ \\
& 1.5 & 1.4 & 1.2 & 0.0010 & $1.0 \mathrm{e}-06$ & $1.5 \mathrm{e}-06$ & $3.7 \mathrm{e}-06$ & $3.9 \mathrm{e}-06$ & $7.2 \mathrm{e}-07$ & $2.9 \mathrm{e}-06$ & $1.4 \mathrm{e}-05$ & 0.00011 & $8.3 \mathrm{e}-06$ \\
\hline 100 & 0.5 & 0.4 & 0.8 & 0.0012 & $3.0 \mathrm{e}-06$ & $7.1 \mathrm{e}-07$ & $5.6 \mathrm{e}-06$ & $3.9 \mathrm{e}-06$ & $2.5 \mathrm{e}-06$ & $5.2 \mathrm{e}-08$ & $2.5 \mathrm{e}-05$ & $3.3 \mathrm{e}-05$ & $2.3 \mathrm{e}-06$ \\
& 0.5 & 0.4 & 1.2 & 0.0015 & $5.4 \mathrm{e}-06$ & $8.2 \mathrm{e}-07$ & $5.0 \mathrm{e}-06$ & $9.9 \mathrm{e}-06$ & $3.8 \mathrm{e}-06$ & $3.6 \mathrm{e}-07$ & $1.4 \mathrm{e}-05$ & 0.00013 & $5.8 \mathrm{e}-06$ \\
& 0.5 & 1.4 & 0.8 & 0.0012 & $5.3 \mathrm{e}-06$ & $1.5 \mathrm{e}-06$ & $7.7 \mathrm{e}-06$ & $6.2 \mathrm{e}-06$ & $4.0 \mathrm{e}-06$ & $1.0 \mathrm{e}-06$ & $3.2 \mathrm{e}-05$ & $4.7 \mathrm{e}-05$ & $5.4 \mathrm{e}-06$ \\
& 0.5 & 1.4 & 1.2 & 0.0012 & $3.5 \mathrm{e}-06$ & $2.7 \mathrm{e}-07$ & $4.1 \mathrm{e}-06$ & $7.4 \mathrm{e}-06$ & $2.7 \mathrm{e}-06$ & $7.7 \mathrm{e}-07$ & $1.2 \mathrm{e}-05$ & 0.00011 & $2.4 \mathrm{e}-06$ \\
& 1.5 & 0.4 & 0.8 & 0.0006 & $5.6 \mathrm{e}-07$ & $1.1 \mathrm{e}-07$ & $2.9 \mathrm{e}-06$ & $1.4 \mathrm{e}-06$ & $8.5 \mathrm{e}-07$ & $7.8 \mathrm{e}-07$ & $1.5 \mathrm{e}-05$ & $1.6 \mathrm{e}-05$ & $7.4 \mathrm{e}-07$ \\
& 1.5 & 0.4 & 1.2 & 0.0009 & $1.3 \mathrm{e}-06$ & $1.8 \mathrm{e}-07$ & $2.5 \mathrm{e}-06$ & $4.0 \mathrm{e}-06$ & $1.3 \mathrm{e}-06$ & $9.1 \mathrm{e}-07$ & $8.3 \mathrm{e}-06$ & $7.1 \mathrm{e}-05$ & $5.2 \mathrm{e}-07$ \\
& 1.5 & 1.4 & 0.8 & 0.0007 & $2.9 \mathrm{e}-06$ & $7.2 \mathrm{e}-07$ & $5.3 \mathrm{e}-06$ & $3.7 \mathrm{e}-06$ & $2.4 \mathrm{e}-06$ & $1.5 \mathrm{e}-07$ & $2.3 \mathrm{e}-05$ & $3.1 \mathrm{e}-05$ & $2.4 \mathrm{e}-06$ \\
& 1.5 & 1.4 & 1.2 & 0.0009 & $5.0 \mathrm{e}-07$ & $7.1 \mathrm{e}-07$ & $1.7 \mathrm{e}-06$ & $1.7 \mathrm{e}-06$ & $3.2 \mathrm{e}-07$ & $1.3 \mathrm{e}-06$ & $6.4 \mathrm{e}-06$ & $5.1 \mathrm{e}-05$ & $3.7 \mathrm{e}-06$ \\
\hline
\end{tabular}

In Table 4.6 we present the Absolute Bias estimated values for the shape parameter $\boldsymbol{\beta}$ for the MLE and Bayesian Estimation using Jeffreys prior information with the three loss functions.

Table 4.8:- Absolute Bias Estimated Parameter $(\boldsymbol{\beta})$ using Jeffreys prior information

\begin{tabular}{|c|c|c|c|c|c|c|c|c|c|c|c|}
\hline \multirow[b]{2}{*}{$\mathrm{n}$} & \multirow[b]{2}{*}{$\sigma$} & \multirow[b]{2}{*}{$\beta$} & \multirow[b]{2}{*}{$\hat{\beta}_{B S}$} & $\hat{\beta}_{B L}$ & $\hat{\beta}_{B G}$ & $\hat{\beta}_{B L}$ & $\hat{\beta}_{B G}$ & $\hat{\beta}_{B L}$ & $\hat{\beta}_{B G}$ & $\hat{\beta}_{B L}$ & $\hat{\beta}_{B G}$ \\
\hline & & & & \multicolumn{2}{|c|}{$\mathrm{a}=\mathrm{k}=0.6$} & \multicolumn{2}{|c|}{$\mathrm{a}=\mathrm{k}=-0.6$} & \multicolumn{2}{|c|}{$\mathrm{a}=\mathrm{k}=1.6$} & \multicolumn{2}{|c|}{$\mathrm{a}=\mathrm{k}=-1.6$} \\
\hline \multirow[t]{8}{*}{25} & 0.5 & 0.8 & $1.2 \mathrm{e}-05$ & $3.0 \mathrm{e}-06$ & $2.2 \mathrm{e}-05$ & $1.5 \mathrm{e}-05$ & $1.0 \mathrm{e}-05$ & $6.0 \mathrm{e}-07$ & $9.9 \mathrm{e}-05$ & 0.00013 & $1.0 \mathrm{e}-05$ \\
\hline & 0.5 & 1.2 & $1.8 \mathrm{e}-05$ & $2.6 \mathrm{e}-06$ & $1.8 \mathrm{e}-05$ & $3.5 \mathrm{e}-05$ & $1.3 \mathrm{e}-05$ & $1.8 \mathrm{e}-06$ & $5.4 \mathrm{e}-05$ & 0.00049 & $1.8 \mathrm{e}-05$ \\
\hline & 0.5 & 0.8 & $9.3 e-06$ & $2.0 \mathrm{e}-06$ & $1.9 \mathrm{e}-05$ & $1.2 \mathrm{e}-05$ & $8.3 \mathrm{e}-06$ & $5.2 \mathrm{e}-07$ & $8.8 \mathrm{e}-05$ & 0.00011 & $6.1 \mathrm{e}-06$ \\
\hline & 0.5 & 1.2 & $2.1 \mathrm{e}-05$ & $3.1 \mathrm{e}-06$ & $2.0 \mathrm{e}-05$ & $4.0 \mathrm{e}-05$ & $1.5 \mathrm{e}-05$ & $1.7 \mathrm{e}-06$ & $6.1 \mathrm{e}-05$ & 0.00055 & $2.2 \mathrm{e}-05$ \\
\hline & 1.5 & 0.8 & $5.6 \mathrm{e}-06$ & $2.7 \mathrm{e}-07$ & $1.8 \mathrm{e}-05$ & $1.0 \mathrm{e}-05$ & $6.4 \mathrm{e}-06$ & $3.3 \mathrm{e}-06$ & $9.2 \mathrm{e}-05$ & 0.00010 & $6.7 \mathrm{e}-07$ \\
\hline & 1.5 & 1.2 & $4.0 \mathrm{e}-06$ & $8.2 \mathrm{e}-07$ & $8.6 \mathrm{e}-06$ & $1.3 \mathrm{e}-05$ & $4.3 \mathrm{e}-06$ & $3.3 \mathrm{e}-06$ & $2.8 \mathrm{e}-05$ & 0.00024 & $3.0 \mathrm{e}-06$ \\
\hline & 1.5 & 0.8 & $1.4 \mathrm{e}-06$ & $2.8 \mathrm{e}-07$ & $7.4 \mathrm{e}-06$ & $3.5 \mathrm{e}-06$ & $2.1 \mathrm{e}-06$ & $1.9 \mathrm{e}-06$ & $3.7 \mathrm{e}-05$ & $4.1 \mathrm{e}-05$ & $1.8 \mathrm{e}-06$ \\
\hline & 1.5 & 1.2 & $2.0 \mathrm{e}-06$ & $8.0 \mathrm{e}-07$ & $5.6 \mathrm{e}-06$ & $8.4 \mathrm{e}-06$ & $2.6 \mathrm{e}-06$ & $2.5 \mathrm{e}-06$ & $1.9 \mathrm{e}-05$ & 0.00016 & $3.4 \mathrm{e}-06$ \\
\hline \multirow[t]{8}{*}{50} & 0.5 & 0.8 & $3.2 \mathrm{e}-06$ & $6.2 \mathrm{e}-07$ & $7.2 \mathrm{e}-06$ & $4.6 \mathrm{e}-06$ & $2.9 \mathrm{e}-06$ & $4.0 \mathrm{e}-07$ & $3.3 \mathrm{e}-05$ & $4.2 \mathrm{e}-05$ & $1.8 \mathrm{e}-06$ \\
\hline & 0.5 & 1.2 & $9.6 \mathrm{e}-06$ & $1.3 \mathrm{e}-06$ & $9.5 \mathrm{e}-06$ & $1.8 \mathrm{e}-05$ & 7.0e-06 & $9.9 \mathrm{e}-07$ & $2.8 \mathrm{e}-05$ & 0.00025 & $9.5 \mathrm{e}-06$ \\
\hline & 0.5 & 0.8 & $3.3 \mathrm{e}-06$ & $5.8 \mathrm{e}-07$ & $7.9 \mathrm{e}-06$ & $4.9 \mathrm{e}-06$ & $3.1 \mathrm{e}-06$ & $6.1 \mathrm{e}-07$ & $3.7 \mathrm{e}-05$ & $4.6 \mathrm{e}-05$ & $1.5 \mathrm{e}-06$ \\
\hline & 0.5 & 1.2 & $1.0 \mathrm{e}-05$ & $1.7 \mathrm{e}-06$ & $9.8 \mathrm{e}-06$ & $1.9 \mathrm{e}-05$ & $7.6 e-06$ & $5.3 e-07$ & $2.8 \mathrm{e}-05$ & 0.00026 & $1.2 \mathrm{e}-05$ \\
\hline & 1.5 & 0.8 & $2.4 \mathrm{e}-06$ & $6.0 \mathrm{e}-08$ & $8.6 \mathrm{e}-06$ & $4.6 \mathrm{e}-06$ & $2.8 \mathrm{e}-06$ & $1.6 \mathrm{e}-06$ & $4.2 \mathrm{e}-05$ & $4.9 \mathrm{e}-05$ & $5.7 \mathrm{e}-07$ \\
\hline & 1.5 & 1.2 & $1.5 \mathrm{e}-06$ & $4.4 \mathrm{e}-07$ & $3.7 \mathrm{e}-06$ & $5.6 \mathrm{e}-06$ & $1.8 \mathrm{e}-06$ & $1.5 \mathrm{e}-06$ & $1.2 \mathrm{e}-05$ & 0.00010 & $1.8 \mathrm{e}-06$ \\
\hline & 1.5 & 0.8 & $1.3 e-06$ & $7.5 \mathrm{e}-08$ & $5.5 \mathrm{e}-06$ & $3.2 \mathrm{e}-06$ & $2.0 \mathrm{e}-06$ & $1.5 \mathrm{e}-06$ & $3.3 \mathrm{e}-05$ & $3.7 \mathrm{e}-05$ & $1.1 \mathrm{e}-06$ \\
\hline & 1.5 & 1.2 & $2.2 \mathrm{e}-06$ & $5.9 \mathrm{e}-07$ & $5.2 \mathrm{e}-06$ & $8.0 \mathrm{e}-06$ & $2.5 \mathrm{e}-06$ & $2.1 \mathrm{e}-06$ & $1.7 \mathrm{e}-05$ & 0.00014 & $2.3 e-06$ \\
\hline \multirow[t]{8}{*}{100} & 0.5 & 0.8 & $3.2 \mathrm{e}-06$ & $7.8 \mathrm{e}-07$ & $5.8 \mathrm{e}-06$ & $4.1 \mathrm{e}-06$ & $2.6 \mathrm{e}-06$ & $1.3 \mathrm{e}-07$ & $2.5 \mathrm{e}-05$ & $3.5 \mathrm{e}-05$ & $2.5 \mathrm{e}-06$ \\
\hline & 0.5 & 1.2 & $5.1 \mathrm{e}-06$ & $7.4 \mathrm{e}-07$ & $4.9 \mathrm{e}-06$ & $9.5 \mathrm{e}-06$ & $3.6 \mathrm{e}-06$ & $4.2 \mathrm{e}-07$ & $1.4 \mathrm{e}-05$ & 0.00013 & $5.3 e-06$ \\
\hline & 0.5 & 0.8 & 3.0e- -06 & $7.0 \mathrm{e}-07$ & $5.7 \mathrm{e}-06$ & $4.0 \mathrm{e}-06$ & $2.5 \mathrm{e}-06$ & $2.4 \mathrm{e}-08$ & $2.5 \mathrm{e}-05$ & $3.4 \mathrm{e}-05$ & $2.2 \mathrm{e}-06$ \\
\hline & 0.5 & 1.2 & $5.5 \mathrm{e}-06$ & $8.5 \mathrm{e}-07$ & $5.0 \mathrm{e}-06$ & $9.9 \mathrm{e}-06$ & $3.8 \mathrm{e}-06$ & $3.1 \mathrm{e}-07$ & $1.4 \mathrm{e}-05$ & 0.00013 & $6.0 \mathrm{e}-06$ \\
\hline & 1.5 & 0.8 & $3.1 \mathrm{e}-06$ & $5.6 \mathrm{e}-08$ & $3.1 \mathrm{e}-06$ & $3.1 \mathrm{e}-06$ & $9.5 e-07$ & 7.1e-07 & $1.5 \mathrm{e}-05$ & $1.7 \mathrm{e}-05$ & $5.1 \mathrm{e}-07$ \\
\hline & 1.5 & 1.2 & $1.1 \mathrm{e}-06$ & $2.4 \mathrm{e}-07$ & $2.4 \mathrm{e}-06$ & $3.8 \mathrm{e}-06$ & $1.2 \mathrm{e}-06$ & $9.5 \mathrm{e}-07$ & $8.1 \mathrm{e}-06$ & $6.9 \mathrm{e}-05$ & $8.8 \mathrm{e}-07$ \\
\hline & 1.5 & 0.8 & $9.3 \mathrm{e}-07$ & $8.8 \mathrm{e}-07$ & $3.5 \mathrm{e}-06$ & $1.8 \mathrm{e}-06$ & $1.1 \mathrm{e}-06$ & $7.4 \mathrm{e}-07$ & $1.7 \mathrm{e}-05$ & $2.0 \mathrm{e}-05$ & $4.0 \mathrm{e}-07$ \\
\hline & 1.5 & 1.2 & $1.0 \mathrm{e}-06$ & $2.7 \mathrm{e}-07$ & $2.3 \mathrm{e}-06$ & $3.6 \mathrm{e}-06$ & $1.1 \mathrm{e}-06$ & $9.8 \mathrm{e}-07$ & $8.0 \mathrm{e}-06$ & $6.7 \mathrm{e}-05$ & $1.0 \mathrm{e}-06$ \\
\hline
\end{tabular}

Similarly, it has also been observed from Table 4.7 and 4.8 the estimator that gives the minimum Absolute Bias over all the other estimators in majority of the cases is Bayes estimator under LINEX loss function.

\section{Illustration:-}

The real data set is about a clinical Trial in the Treatment of Carcinoma of the Oropharynx (PHARYNX) data extracted from [22]. The data file gives the data for a part of a large clinical trial carried out by the Radiation Therapy Oncology Group in the United States. This data consists of a total of 195 respondents of which 53 are alive and 142 are dead. Here we considered age in years at time of diagnosis is the most factors. Table 5.1 depicts the Standard Error values for estimated parameters using PHARYNX Data.

Table 5.1:- Estimated parameter Using PHARYNX Data 


\begin{tabular}{|c|c|c|c|c|}
\hline Parameters & MLE & BS & BL & BG \\
\hline$\hat{\sigma}$ & 1.0206 & 0.5000 & 0.0997 & 24.0223 \\
\hline$\hat{\beta}$ & 0.9969 & 0.5971 & 0.1997 & 24.1045 \\
\hline
\end{tabular}

ML: Maximum Likelihood

BS: Squared Error Loss function

BL: LINEX Loss function

BG: General Entropy Loss function.

From Table 5.1, we observe that, Bayesian estimator under LINEX loss function has the smallest values for both the scale parameter $\boldsymbol{\sigma}$ and the shape parameter $\boldsymbol{\beta}$. So that the Bayes estimators of parameters under LINEX loss function is best estimation method for Constant Shape Bi-Weibull Distribution using PHARYNX Data.

\section{Conclusion:-}

In this paper, we have addressed the problem of Bayesian estimation for the Constant Shape Bi-Weibull distribution, under Asymmetric and Symmetric loss functions and that of Maximum Likelihood Estimation. Bayes estimators were obtained using Lindley approximation while MLE were obtained using Newton-Raphson method. A Simulation study was conducted to examine and compare the performance of the estimates for different sample sizes with different values for the extension of Jeffreys' prior and the loss functions. From the results, we observe that in most cases, Bayesian estimator under LINEX loss function has the smallest Mean Squared Error values and minimum Bias for both the scale parameter $\boldsymbol{\sigma}$ and the shape parameter $\boldsymbol{\beta}$ in most cases especially compared when the loss parameter values are $(0.6,1.6)$, for both values of the extension of Jeffreys' prior and Jeffreys' information.

\section{References:-}

1. Abdel-Wahid, A. and Winterbottom, A. (1987). Approximate Bayesian Estimates for the Weibull Reliability Function and Hazard Rate from Censored Data, J. Stat Plann and Infer, 16, 277-283.

2. Al-Aboud, F. M. (2009). Bayesian Estimation for the Extreme Value Distribution Using Progressive Censored Data and Asymmetric Loss, International Mathematical Forum, 4, 1603-1622.

3. Al-Athari, F. M. (2011). Parameter Estimation for the Double-Pareto Distribution, J. Math Stat, 7, 289-294.

4. Al Omari, M. A. and Ibrahim, N. A. (2011). Bayesian Survival Estimation for Weibull Distribution with Censored Data, J. Appl Sci, 11, 393-396.

5. Calabria, R. and Pulcini, G. (1996). Point Estimation under Asymmetric Loss Function for Left Truncated Exponential Samples, Comm Stat Theor Meth, 25, 585-600.

6. Guure, C. B., Ibrahim, N. A. and Adam, M. B. (2013). Bayesian Inference of the Weibull Model Based on Interval-Censored Survival Data. Comp Math Methods Med, Article ID 849520, 10 pages.

7. Guure, C. B. and Ibrahim, N.A. (2012a). Bayesian analysis of the survival function and failure rate of Weibull distribution with censored data, Math Probl Eng, Article ID. 329489, 18 pages.

8. Guure, C. B., Ibrahim, N. A. and Al Omari, A. M. (2012). Bayesian Estimation of Two-Parameter Weibull Distribution using Extension of Jeffreys' Prior Information with Three Loss Functions. Math Probl Eng., Article ID. 589640.13 pages.

9. Lavanya, A. and Leo Alexander, T. (2016). Confidence Intervals Estimation for ROC Curve, AUC and Brier Score under the Constant Shape Bi-Weibull Distribution, International Journal of Science and Research, 5, 8, 371-378.

10. Lavanya, A. and Leo Alexander, T. (2016). Asymmetric and Symmetric Properties of Constant Shape BiWeibull ROC Curve Described by Kullback-Leibler Divergences, International Journal of Applied Research, 2, 8, 713-720.

11. Lavanya, A. and Leo Alexander, T. (2016). Bayesian Estimation of Parameters under the Constant Shape BiWeibull Distribution Using Extension of Jeffreys' Prior Information with Three Loss Functions, International Journal of Science and Research, 5, 9, 96-103.

12. Lavanya, A. and Leo Alexander, T. (2016). Bayesian Estimation of the Failure Rate Using Extension of Jeffreys Prior Information with Three Loss Functions, International Journal of Science and Research, 5, 9, 736-742.

13. Lavanya, A. and Leo Alexander, T. (2016). Estimation Of The Survival Function Under The Constant Shape Bi-Weibull Failure Time Distribution Based On Three Loss Functions, International Journal of advanced Research, 4, 9, 1225-1234.

14. Leo Alexander, T. and Lavanya, A. (2016). Functional Relationship between Brier Score and Area Under the Constant Shape Bi-Weibull ROC Curve, International Journal of Recent Scientific Research 7, 7, 12330-12336. 
15. Leo Alexander, T. and Lavanya, A. (2016). Estimation Of AUC For Constant Shape Bi-Weibull Failure Time Distribution, International Journal of Recent Scientific Research, 7, 10, 13826-13836.

16. Pandey, B. N., Dwividi, N. and Pulastya, B. (2011). Comparison Between Bayesian and Maximum Likelihood Estimation of the Scale Parameter in Weibull Distribution with Known Shape Under Linex Loss Function, J. Sci Res, 55, 163-172.

17. Sinha, S. k. and Sloan, J.A. (1988). Bayes Estimation of the Parameters and Reliability Function of the 3Parameter Weibull Distribution, IEEE Transactions on Reliability, 37, 364-369.

18. Sinha S. K. (1986). Bayes Estimation of the Reliability Function and Hazard Rate of a Weibull Failure Time Distribution, Tranbajos De Estadistica, 1, 47-56.

19. Soliman, A. A., Abd Ellah, A. H. and Ryan, S. B. (2006). Comparison of Estimates Using Record Statistics From Weibull Model: Bayesian and Non-Bayesian Approaches. Comput Stat Data Anal, 51, 2065-2077.

20. Syuan-Rong, H. and Shuo-Jye W. (2011). Bayesian Estimation and Prediction for Weibull Model with Progressive Censoring, J. Stat Comput Simulat, 1-14.

21. Zellner, A. (1986). Bayesian Estimation and Prediction Using Asymmetric Loss Functions, J. Am Stat Assoc, $81,446-451$.

22. www.umass.edu/statdata/statdata/data/pharynx.txt 